\title{
Cosmic ray induced ionisation of a molecular cloud shocked by the W28 supernova remnant
}

\author{
S. Vaupré ${ }^{1}$, P. Hily-Blant ${ }^{1}$, C. Ceccarelli ${ }^{1}$, G. Dubus ${ }^{1}$, S. Gabici ${ }^{2}$, and T. Montmerle ${ }^{3}$ \\ 1 Univ. Grenoble Alpes/CNRS, IPAG, 38000 Grenoble, France \\ e-mail: solenn. vaupre@obs.ujf-grenoble.fr \\ 2 APC, AstroParticule et Cosmologie, Université Paris Diderot, CNRS, CEA, Observatoire de Paris, Sorbonne Paris, 75205 Paris, \\ France \\ ${ }^{3}$ Institut d'Astrophysique de Paris, 98bis Bd Arago, 75014 Paris, France
}

Received 19 April 2014 / Accepted 5 June 2014

\section{ABSTRACT}

\begin{abstract}
Cosmic rays are an essential ingredient in the evolution of the interstellar medium, as they dominate the ionisation of the dense molecular gas, where stars and planets form. However, since they are efficiently scattered by the galactic magnetic fields, many questions remain open, such as where exactly they are accelerated, what is their original energy spectrum, and how they propagate into molecular clouds. In this work we present new observations and discuss in detail a method that allows us to measure the cosmic ray ionisation rate towards the molecular clouds close to the W28 supernova remnant. To perform these measurements, we use $\mathrm{CO}, \mathrm{HCO}^{+}$, and $\mathrm{DCO}^{+}$millimetre line observations and compare them with the predictions of radiative transfer and chemical models away from thermodynamical equilibrium. The $\mathrm{CO}$ observations allow us to constrain the density, temperature, and column density towards each observed position, while the $\mathrm{DCO}^{+} / \mathrm{HCO}^{+}$abundance ratios provide us with constraints on the electron fraction and, consequently, on the cosmic ray ionisation rate. Towards positions located close to the supernova remnant, we find cosmic ray ionisation rates much larger $(\gtrsim 100)$ than those in standard galactic clouds. Conversely, towards one position situated at a larger distance, we derive a standard cosmic ray ionisation rate. Overall, these observations support the hypothesis that the $\gamma$ rays observed in the region have a hadronic origin. In addition, based on CR diffusion estimates, we find that the ionisation of the gas is likely due to $0.1-1 \mathrm{GeV}$ cosmic rays. Finally, these observations are also in agreement with the global picture of cosmic ray diffusion, in which the low-energy tail of the cosmic ray population diffuses at smaller distances than the high-energy counterpart.
\end{abstract}

Key words. ISM: clouds - cosmic rays - ISM: supernova remnants - ISM: individual objects: W28

\section{Introduction}

Cosmic rays (CRs) are energetic charged particles that reach the Earth as an isotropic flux. They pervade the Galaxy and play a crucial role in the evolution of the interstellar medium, because they dominate the ionisation of molecular clouds where the gas is shielded from the UV radiation field. The ionisation degree in the molecular gas is a fundamental parameter throughout the star and planet forming process. First, ions couple the gas to the magnetic field and, therefore, they regulate the gravitational collapse of the cloud. In addition, ions sustain turbulence within protoplanetary discs and introduce non-ideal magnetohydrodynamics effects, which influence the accretion rate onto the protostar (Balbus \& Hawley 1998; Lesur et al. 2014). Finally, the $\mathrm{CR}$ induced ions initiate efficient chemical reactions in the cold molecular clouds, which eventually lead to the formation of complex molecules, which enrich the gas even up to the first stages of planet formation.

However, in order to fully understand the influence of CRs on the above processes across the Galaxy, it is necessary to know where CRs are accelerated and how they propagate through the gas. Unfortunately, since CRs are scattered by magnetic fields all along their path through the Galaxy, the production sites of CRs cannot be observed directly, and the diffusion of CRs also makes the evolution of the energy spectrum during their propagation difficult to determine observationally.

However, we can detect indirect signatures of the interaction of hadronic CRs (essentially protons) with matter. Protons above a kinetic energy threshold of $\approx 280 \mathrm{MeV}$ produce $\pi^{0}$ pions when they collide with particles in the molecular cloud. Each pion then decays into two $\gamma$-ray photons $\left(\pi^{0} \rightarrow 2 \gamma\right)$, each with a typical energy that thoseis $\sim 10 \%$ that of the colliding proton. Bright $\gamma$-ray sources thus indicate regions with a large density of protons with energies above $0.28 \mathrm{GeV}$. In these regions, the observed $\gamma$-ray photon spectrum can, in addition, be used to derive the spectrum of the parental CR particles, before the scattering and propagation within the Galaxy (Ackermann et al. 2013).

Supernova remnants (SNR) are thought to be the sources of CRs. In this scenario, protons are accelerated in the expanding shell of the SNR, following the diffusive shock acceleration process (Bell 1978). Supporting this scenario, there is now clear evidence that SNR are spatially associated with $\mathrm{GeV}$ to $\mathrm{TeV}$ sources (Aharonian 2013). Moreover, several SNR are close to the molecular cloud that gave birth to the SN precursor. These molecular clouds now act as reservoirs of target material for the freshly accelerated protons, thus enhancing the production rate of $\gamma$ rays. Although it is compatible with $\gamma$-ray observations, the hadronic scenario is challenged by the leptonic scenario involving electron CRs. In this alternative scenario, the $\gamma$-ray emission can be explained mainly by inverse Compton scattering of the cosmic microwave background (e.g. Morlino et al. 2009; Abdo et al. 2011). Yet, this scenario cannot explain the spatial correlation of $\mathrm{TeV}$ emission with molecular clouds. Moreover, recent observations of the IC443 and W44 SNR with the Fermi-LAT telescope (Ackermann et al. 2013) specifically 

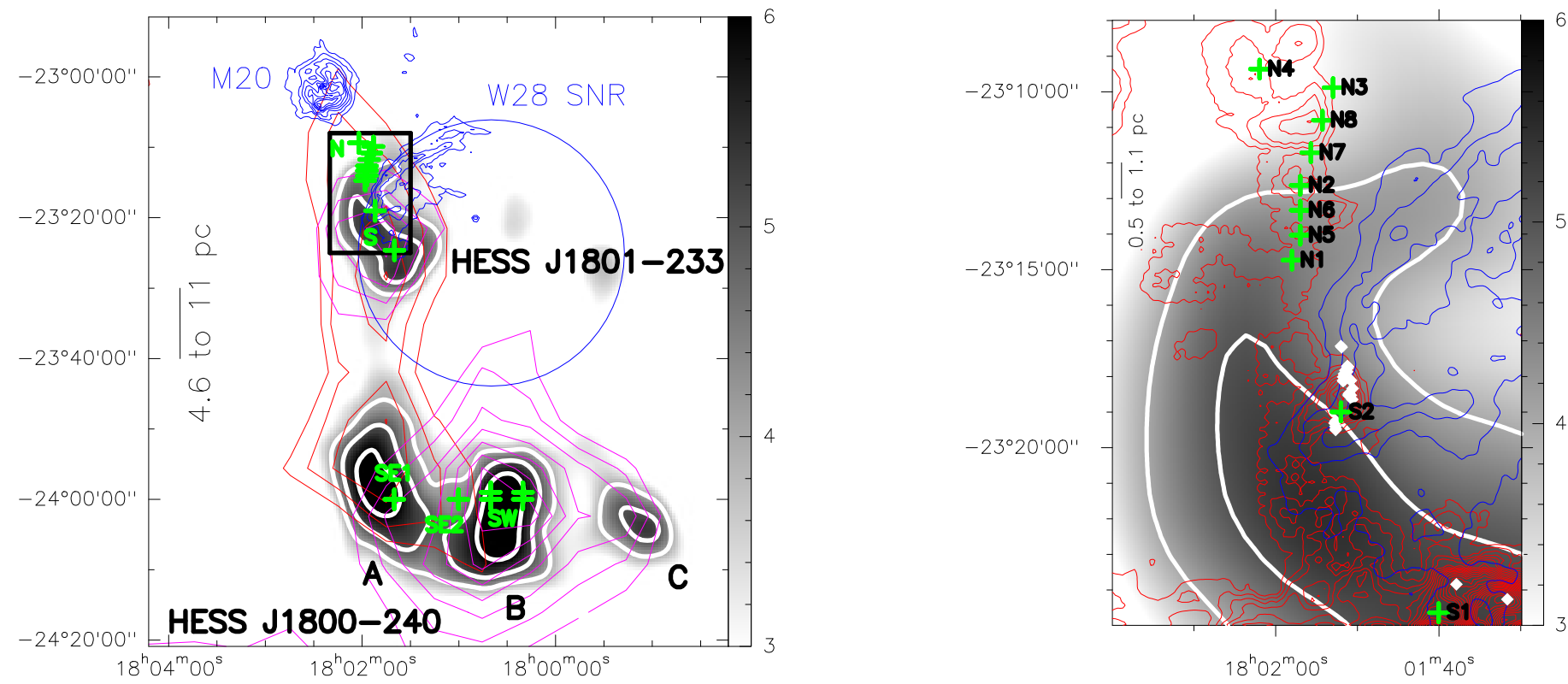

Fig. 1. Left: the W28 complex on large scales. Grayscale (in $\sigma$ ) and thick contours show TeV emission as seen by HESS (levels are 4-6 $\sigma$ ). Red contours show the $\mathrm{CO}(1-0)$ emission (Dame et al. 2001) integrated over $15-25 \mathrm{~km} \mathrm{~s}^{-1}$ and magenta contours trace the emission integrated over $5-15 \mathrm{~km} \mathrm{~s}^{-1}$ (levels are $40-70 \mathrm{~K} \mathrm{~km} \mathrm{~s}^{-1}$ by $5 \mathrm{~K} \mathrm{~km} \mathrm{~s}^{-1}$ ). Crosses show the positions observed with the IRAM 30m telescope and discussed in this paper. The blue contours show the $20 \mathrm{~cm}$ free-free emission in the M20 region (Yusef-Zadeh et al. 2000). The blue circle gives the approximate radio boundary of the SNR W28 (Brogan et al. 2006). Right: the northern cloud in the W28 complex (zoom on the black box). The red contours show the $\mathrm{CO}(3-2)$ emission in $\mathrm{K} \mathrm{km} \mathrm{s}^{-1}$, integrated over $15-25 \mathrm{~km} \mathrm{~s}^{-1}$ (levels are $15-130 \mathrm{~K} \mathrm{~km} \mathrm{~s}^{-1}$ by $5 \mathrm{~K} \mathrm{~km} \mathrm{~s}^{-1}$ ) (Lefloch et al. 2008). Diamonds show the locations of OH masers in the region (Claussen et al. 1997).

support a hadronic origin of $\gamma$ rays, consistent with the so-called SNR paradigm for the origin of primary CR (see e.g. Hillas 2005, for a review).

Cosmic ray protons with kinetic energy below the $\approx 280 \mathrm{MeV}$ threshold of $\pi^{0}$ production cannot be traced by the emission of $\gamma$-rays. Nevertheless, recent calculations suggest that the ionisation of UV-shielded gas is mostly due to keV-GeV protons (Padovani et al. 2009). Accordingly, low-energy CR protons can be traced indirectly by measuring the ionisation fraction of the dense gas. It has thus been proposed that an enhanced electron abundance in molecular clouds located in the vicinity of SNR could be the smoking gun for the presence of freshly accelerated CRs, with energies $\lesssim 1 \mathrm{GeV}$.

This idea was put forward by Ceccarelli et al. (2011, hereafter CC2011), who measured the ionisation fraction $x_{\mathrm{e}}=n\left(\mathrm{e}^{-}\right) / n_{\mathrm{H}}$ in the W51C molecular cloud, located in the vicinity of the W51 SNR. The detection of TeV emission by both HESS and MAGIC telescopes close to the molecular cloud is evidence of a physical interaction with the SNR. This supports the idea of the pion-decay production of $\gamma$ rays with W51C acting as a $\gamma$-ray emitter. In CC2011, an enhanced ionisation fraction was reported towards one position, W51C-E, which required a CR ionisation rate two orders of magnitude larger than the typical value of $1 \times 10^{-17} \mathrm{~s}^{-1}$ in molecular clouds. This observational evidence strongly supports the hadronic scenario of $\gamma$-ray production, at least for W51.

Complementary studies of the CR ionisation rate in several diffuse clouds close to SNR have been carried out using different techniques, such as $\mathrm{H}_{3}^{+}$absorption (McCall et al. 2003). These studies also show an enhancement of a factor of 10-100 of the CR ionisation rate (Indriolo et al. 2010; Indriolo \& McCall 2012) with respect to the canonical value. However, the interpretation is not straightforward, as Padovani et al. (2009) showed that the penetration into the cloud of high energy CRs results into an enhanced CR ionisation rate in low density molecular clouds even in absence of an increased CR flux.

The combined observations of two extreme energy ranges, namely $\mathrm{TeV}$ and millimetre, seems a powerful method to characterise an enhanced concentration of proton CRs. It also gives additional evidence supporting a physical interaction of the SNR shock with molecular clouds. From a theoretical point of view, it is expected that the most energetic CR protons diffuse at larger distances ahead of the SNR shock front, whilst the low-energy tail of the distribution remains closer. As a consequence, one expects that any ionisation enhancement by low energy CRs should be localised accordingly. In CC2011, however, only one location could be used to derive the ionisation fraction, and no constraint could be given regarding the spatial distribution of the ionisation and therefore the diffusion properties of CRs.

The aim of this paper is to present measurements of the ionisation fraction within the molecular clouds in the vicinity of the W28 SNR. The paper is organized as follows. In Sect. 2, the W28 association is presented, with particular emphasis on the physical link between the SNR and the molecular clouds. In Sect. 3, the millimetre observations are described. The derivation of the physical conditions is presented in Sect. 4. The derivation of the ionisation fraction and the CR ionisation rates are described in Sects. 5 and 6, where we stress the strengths and limitations of the method. The results are discussed in Sect. 7.

\section{The W28 association}

The W28 SNR has an age greater than $10^{4} \mathrm{yr}$, and is likely in the Sedov or radiative phase (Westerhout 1958; Lozinskaya 1974). Its distance is estimated between $1.6 \mathrm{kpc}$ and $4 \mathrm{kpc}$, based on kinematic determinations and $\mathrm{H} \alpha$ observations (Goudis 1976; Lozinskaya 1981). The LSR velocity, based on $\mathrm{H} \alpha$ and [NII] observations, is estimated to be $18 \pm 5 \mathrm{~km} \mathrm{~s}^{-1}$ (Lozinskaya 1974). 
Table 1. Molecular transitions observed with the IRAM $30 \mathrm{~m}$ telescope.

\begin{tabular}{lcrccccc}
\hline \hline Species & Line & $\begin{array}{r}\text { Frequency } \\
{[\mathrm{GHz}]}\end{array}$ & $F_{\text {eff }}$ & $B_{\text {eff }}$ & $\begin{array}{r}H P B W \\
{[\operatorname{arcsec}]}\end{array}$ & $\begin{array}{c}T_{\text {sys }} \\
{[\mathrm{K}]}\end{array}$ & $\begin{array}{c}\sigma_{\text {rms }} \\
{[\mathrm{mK}]}\end{array}$ \\
\hline $\mathrm{H}^{13} \mathrm{CO}^{+}$ & $(1-0)$ & 86.754 & 0.95 & 0.81 & 29 & $100-130$ & $6-12$ \\
$\mathrm{C}^{18} \mathrm{O}$ & $(1-0)$ & 109.782 & 0.95 & 0.79 & 22 & $140-200$ & $20-50$ \\
${ }^{13} \mathrm{CO}$ & $(1-0)$ & 110.201 & 0.95 & 0.79 & 22 & $140-200$ & $20-50$ \\
$\mathrm{C}^{17} \mathrm{O}$ & $(1-0)$ & 112.359 & 0.95 & 0.79 & 22 & $140-200$ & $20-50$ \\
$\mathrm{DCO}^{+}$ & $(2-1)$ & 144.077 & 0.92 & 0.74 & 16 & $100-200$ & $8-20$ \\
$\mathrm{C}^{18} \mathrm{O}$ & $(2-1)$ & 219.560 & 0.94 & 0.61 & 11 & $160-220$ & $20-80$ \\
${ }^{13} \mathrm{CO}$ & $(2-1)$ & 220.399 & 0.94 & 0.61 & 11 & $160-220$ & $20-80$ \\
$\mathrm{C}^{17} \mathrm{O}$ & $(2-1)$ & 224.714 & 0.94 & 0.61 & 11 & $160-220$ & $20-80$ \\
\hline
\end{tabular}

Notes. $T_{\text {sys }}$ indicates the range of system temperatures during the observing run, and the corresponding sensitivity fluctuations. The adopted values of the telescope parameters follow from the IRAM observatory recommendations: $F_{\text {eff }}$ and $B_{\text {eff }}$ are the forward and main-beam efficiencies of the telescope, respectively; HPBW is the half-power beam width.

In the remainder of the text, all velocities will refer to the local standard of rest (LSR) and projected distances will be given for distances of both 1.6 and $4 \mathrm{kpc}$. The boundary diameter of the SNR is 42 arcmin, corresponding to a linear radius of 9.6 to 24 pc (Fig. 1).

The large-scale region towards W28 contains a variety of objects such as HII regions (e.g. M8, M20, W28A2), molecular clouds, SNR, and new SNR candidates (Brogan et al. 2006). Molecular gas, as seen in CO(1-0) (Wootten 1981; Dame et al. 2001), coincides spatially with the W28 SNR, suggesting a physical association, and supporting a view in which the W28 SNR is interacting with its parental molecular cloud. Probably related is the fact that ongoing massive star formation has been observed in these molecular clouds, consistent with the triggered star formation scenario (Elmegreen 1998). The molecular gas located towards the north-east of the SNR boundary was mapped at high spatial resolution, in the $\mathrm{CO}(3-2)$ rotational line, by Lefloch et al. (2008) revealing a fragmented filamentary structure elongated north-south (Fig. 1).

In $\gamma$ rays, the high spatial resolution H.E.S.S. imaging array of Cherenkov telescopes has revealed the presence of extended $\mathrm{TeV}$ emission (Aharonian et al. 2008), which splits into two components, separated by 14-34 pc: HESS J1801-233, in the north, and HESS J1800-240 in the south. The latter further splits into three well-separated components (Fig. 1). In projection, the entire $\mathrm{TeV}$ emission coincides with the molecular gas seen in $\mathrm{CO}(1-0)$ which also appears to bridge the northern and southern $\mathrm{TeV}$ components. The molecular gas coinciding with the northern $\mathrm{TeV}$ component J1801-233 shows velocities predominantly from $15 \mathrm{~km} \mathrm{~s}^{-1}$ to $25 \mathrm{~km} \mathrm{~s}^{-1}$, as does the southern J1801-240 A component observed in CS(1-0) by Nicholas et al. (2012). However, the southern components J1801-240 B and $\mathrm{C}$ coincide with molecular emission characterised by somewhat lower velocities, from $5 \mathrm{~km} \mathrm{~s}^{-1}$ to $15 \mathrm{~km} \mathrm{~s}^{-1}$.

Whether the $\mathrm{TeV}$ emission is physically associated with the molecular clouds is of utmost importance, for the question of pion-decay production. However, there are several indications, based on kinematic information, that this may well be the case.

First, when inspected in velocity space, the $\mathrm{CO}(1-0)$ emission covers the entire range from 5 to $25 \mathrm{~km} \mathrm{~s}^{-1}$ continuously (Fukui et al. 2012a). This indicates that the molecular emission, traced either by $\mathrm{CS}$ or $\mathrm{CO}$, is physically linked over the entire region, and not only in projection.

Second, $\mathrm{OH}$ masers have been reported towards the northern component (Claussen et al. 1997; Hewitt et al. 2008), with velocities ranging from $7.1 \mathrm{~km} \mathrm{~s}^{-1}$ to $15.2 \mathrm{~km} \mathrm{~s}^{-1}$. Such OH masers are thought to trace the interaction of the SNR shock with the molecular gas, an interpretation which is consistent with the velocity range of the $\mathrm{CO}(1-0)$ emission.

Finally, velocity differences observed between the northern and southern clouds are compatible with the differences up to $\sim 6 \mathrm{~km} \mathrm{~s}^{-1}$ observed in the M20 map of $\mathrm{CO}(3-2)$, encompassing the northern $\mathrm{TeV}$ component (Lefloch et al. 2008), indicating that velocity shifts of several $\mathrm{km} \mathrm{s}^{-1}$ are found which could be due to an interaction with the SNR.

Taken all together, these data strongly suggest a $3 \mathrm{D}$ picture in which the SNR is interacting with surrounding molecular clouds covering a wide and continuous velocity range, typically from $5 \mathrm{~km} \mathrm{~s}^{-1}$ to $25 \mathrm{~km} \mathrm{~s}^{-1}$, and characterised by large variations along the line of sight.

It is therefore most likely that the W28 association displays the interaction of the SNR with molecular clouds, and thus is an excellent target in which to study the ionisation by CRs.

\section{Observations and data reduction}

Observations were carried out over $40 \mathrm{~h}$ in December, 2011, and March, 2012, with the IRAM 30 m telescope. We used the EMIR bands with the Fast Fourier Transform Spectrometer as a backend in the position-switching mode, using OFF positions about $1000^{\prime \prime}$ to the east. Amplitude calibration was done typically every $15 \mathrm{~min}$, and pointing and focus were checked every 1 and $3 \mathrm{~h}$, respectively, ensuring $\approx 2^{\prime \prime}$ pointing accuracy. All spectra were reduced using the CLASS package of the GILDAS ${ }^{1}$ software (Pety 2005). Residual bandpass effects were subtracted using low-order $(\leq 3)$ polynomials. The weather was good and $T_{\text {sys }}$ values were lower than $220 \mathrm{~K}$. The observed molecular transitions used in the present work are listed in Table 1, along with the associated system temperature and sensitivity ranges $T_{\text {sys }}$ and $\sigma_{\text {rms }}$ obtained during the successive runs of observations. All spectra are presented on the main-beam temperature scale, $T_{\mathrm{mb}}=\left(F_{\text {eff }} / B_{\text {eff }}\right) T_{\text {ant }}^{*}$, with $F_{\text {eff }}$ and $B_{\text {eff }}$ the forward and mainbeam efficiencies of the telescope, respectively.

We observed towards 16 positions, of which 10 are located in the northern cloud and 6 in the southern cloud. The coordinates of these positions are listed in Table 2. The two lowest rotational transitions of ${ }^{13} \mathrm{CO}$ and $\mathrm{C}^{18} \mathrm{O}$ are used to derive the physical conditions in the cloud, while $\mathrm{H}^{13} \mathrm{CO}^{+}(1-0)$ and $\mathrm{DCO}^{+}(2-1)$ are used to derive the cosmic ray ionisation rate.

http://www.iram. fr/IRAMFR/GILDAS/ 
Table 2. J2000 coordinates of the 16 observed positions.

\begin{tabular}{lcc}
\hline \hline Source & $\begin{array}{c}\alpha_{2000} \\
(\mathrm{hms})\end{array}$ & $\begin{array}{c}\delta_{2000} \\
\left({ }^{\prime}{ }^{\prime \prime}\right)\end{array}$ \\
\hline J1801-N1 & 180158.0 & -231444 \\
J1801-N2 $\dagger$ & 180157.0 & -231238 \\
J1801-N3 $\dagger$ & 180153.0 & -230953 \\
J1801-N4 $\dagger$ & 180202.0 & -230922 \\
J1801-N5 & 180157.0 & -231402 \\
J1801-N6 & 180157.0 & -231320 \\
J1801-N7 & 180155.7 & -231143 \\
J1801-N8 & 180154.3 & -231048 \\
J1801-S1 & 180140.0 & -232438 \\
J1801-S2 & 180152.0 & -231900 \\
J1801-SE1 & 180140.0 & -240000 \\
J1801-SE2 & 180100.0 & -240000 \\
J1801-SW1 & 180040.0 & -240000 \\
J1801-SW2 & 180020.0 & -240000 \\
J1801-SW3 & 180040.0 & -235900 \\
J1801-SW4 & 180020.0 & -235900 \\
\hline
\end{tabular}

Notes. $\dagger$ N2, N3, and N4 correspond to TC5, TC7, and TC6, respectively, as referred to in Lefloch et al. (2008). N2 also coincides with a high-mass protostar.

\section{Results}

\subsection{Observed spectra}

The resulting spectra towards all positions are shown in Fig. 2. The ${ }^{13} \mathrm{CO}$ and $\mathrm{C}^{18} \mathrm{O}$ spectra show multiple velocity components, most likely associated with several clouds along the line of sight. In some instances, negative features are apparent, which are due to emission from the reference position at different velocities. Isotopologues ${ }^{13} \mathrm{CO}$ and $\mathrm{C}^{18} \mathrm{O}$ are detected towards 12 of the 16 positions, and the spectra show clearly two main components. The velocity of the dominant component varies between the northern $\left(\lesssim 21 \mathrm{~km} \mathrm{~s}^{-1}\right)$ and southern $\left(\gtrsim 7 \mathrm{~km} \mathrm{~s}^{-1}\right)$ cloud, as presented in Sect. 2. At most positions, the rarer $\mathrm{C}^{17} \mathrm{O}$ isotopologue is also detected, although the hyperfine structure of the $(1-0)$ transition is not always well resolved. The $\mathrm{H}^{13} \mathrm{CO}^{+}(1-0)$ and $\mathrm{DCO}^{+}(2-1)$ emission lines have only one velocity component, at $21 \mathrm{~km} \mathrm{~s}^{-1}$. The $\mathrm{C}^{18} \mathrm{O}(1-0)$ and $(2-1)$ lines show a clear distinction between the northern positions, where the line emission is the strongest, and southern positions. The compound $\mathrm{H}^{13} \mathrm{CO}^{+}$is detected towards all northern positions but $\mathrm{N} 8$. However, $\mathrm{DCO}^{+}$is detected only towards three positions, N2, N5, and N6. Southern positions display much weaker emission and SE1 is detected in both $\mathrm{H}^{13} \mathrm{CO}^{+}$and $\mathrm{DCO}^{+}$.

The analysis was essentially driven by the $\mathrm{H}^{13} \mathrm{CO}^{+}$and $\mathrm{DCO}^{+}$lines which are the main focus of the present work. The velocity of the dominant $\mathrm{CO}$ transition always corresponds to the velocity of the $\mathrm{H}^{13} \mathrm{CO}^{+}$line when detected. When more velocity components are detected in $\mathrm{CO}$, we limited the Gaussian fits to the first two dominant components. Upper limits are given at the $1 \sigma$ level for $T_{\text {peak }}$, and for the integrated intensity $W$ by assuming $\Delta v=3 \mathrm{~km} \mathrm{~s}^{-1}$. The results from the Gaussian fits are summarised in Table 3 (CO isotopologues) and Table $4\left(\mathrm{H}^{13} \mathrm{CO}^{+}\right.$, $\left.\mathrm{DCO}^{+}\right)$.

In the following, we describe the two-step analysis of the measured line intensities. First, the physical conditions are derived according to the ${ }^{13} \mathrm{CO}$ and $\mathrm{C}^{18} \mathrm{O}$ lines. Second, the $\mathrm{HCO}^{+} / \mathrm{DCO}^{+}$abundance ratio is compared with model predictions computed using the derived physical conditions.

\subsection{Determination of physical conditions}

The physical conditions prevailing at the various locations were determined based on the ${ }^{13} \mathrm{CO}(1-0)$ and $(2-1)$ lines and the $\mathrm{C}^{18} \mathrm{O}(1-0)$ and $(2-1)$ lines. To accomplish this, we performed non-local thermal equilibrium (non-LTE) calculations of the rotational level populations under the large velocity gradient (LVG) approximation (Ceccarelli et al. 2003). The $\mathrm{H}_{2}$ density, gas kinetic temperature $T_{\text {kin }}$, and total column density of each species covered a large parameter space. For each set of input parameters, the expected line intensities and integrated intensities were computed adopting the linewidth $\Delta v$ derived from the Gaussian fits (Table 3), and taking into account beam dilution effects by varying the size of the emitting regions. A simple $\chi^{2}$ minimization was then used to constrain the physical conditions that best reproduce the observed intensities. The LVG analysis was performed towards positions where all four lines were detected. We considered collisions of ${ }^{13} \mathrm{CO}$ and $\mathrm{C}^{18} \mathrm{O}$ with both para and ortho $\mathrm{H}_{2}$, assuming an ortho-to-para ratio in Boltzmann equilibrium. Hence, in the temperature range considered here, $\mathrm{H}_{2}$ is mainly in its para configuration. We used the collisional cross sections from Yang et al. (2010).

In this process, the relative abundances of $\mathrm{C}^{18} \mathrm{O}$ to ${ }^{13} \mathrm{CO}$ need to be known, and we have assumed that the molecular isotopic ratios are equal to the elemental isotopic ratios, namely that

$\frac{\left[\mathrm{C}^{18} \mathrm{O}\right]}{\left[{ }^{13} \mathrm{CO}\right]}=\frac{\left[\mathrm{C}^{18} \mathrm{O}\right]}{\left[{ }^{12} \mathrm{C}^{16} \mathrm{O}\right]} \times \frac{\left[{ }^{12} \mathrm{C}^{16} \mathrm{O}\right]}{\left[{ }^{13} \mathrm{CO}\right]} \approx\left(\frac{{ }^{18} \mathrm{O}}{{ }^{16} \mathrm{O}}\right) \times\left(\frac{{ }^{12} \mathrm{C}}{{ }^{13} \mathrm{C}}\right)$.

It is known that isotopic ratios may vary with the position within the Milky Way, resulting from the gradual depletion of ${ }^{12} \mathrm{C}$ and enrichment of ${ }^{13} \mathrm{C}$ with the cycling of gas through stars (e.g. Wilson \& Rood 1994; Frerking et al. 1982). In addition, local variations are also possible, resulting from the competition of chemical fractionation and selective photodissociation (van Dishoeck \& Black 1988; Federman et al. 2003). The dependence of the ${ }^{12} \mathrm{C} /{ }^{13} \mathrm{C}$ isotopic ratio on galactocentric distance was studied by Milam et al. (2005). Applying their results to W28, which is $4-6 \mathrm{kpc}$ from the Galactic center, one gets ${ }^{12} \mathrm{C} /{ }^{13} \mathrm{C}=50 \pm 7$. In practice, in our non-LTE analysis, we varied the ${ }^{13} \mathrm{C} /{ }^{12} \mathrm{C}$ ratio. The best $\chi^{2}$ values were obtained using an isotopic ratio of 50 , consistent with the above expectation, which we therefore adopted in what follows. Regarding the ${ }^{16} \mathrm{O} /{ }^{18} \mathrm{O}$ isotopic ratio, we adopted the value of 500 representative of the solar neighborhood.

The results of the LVG analysis are summarised in Table 5. At each position, the size of the emitting regions was found to be larger than the beam size. Position N2, which is considered a protostar in Lefloch et al. (2008), shows a peculiar behaviour with a visual extinction at least 5 times higher than for the other positions. Overall, the density and kinetic temperature we derived are typical of dense molecular clouds with visual extinctions larger than 10 mag. We also note that the $\mathrm{H}_{2}$ densities we derived in the northern cloud are consistent with values published by Lefloch et al. (2008).

\subsection{The $\mathrm{DCO}^{+} / \mathrm{HCO}^{+}$abundance ratio}

The species $\mathrm{DCO}^{+}$was detected towards four positions, for which it was possible to derive values of the $\mathrm{DCO}^{+} / \mathrm{HCO}^{+}$abundance ratio. For all other positions where $\mathrm{H}^{13} \mathrm{CO}^{+}$was detected, upper limits at the $1 \sigma$ level on the abundance ratio we derived. We determined the column densities of $\mathrm{H}^{13} \mathrm{CO}^{+}$and $\mathrm{DCO}^{+}$from the same non-LTE LVG calculation, using the collisional cross sections from Flower (1999) and the physical conditions derived 
S. Vaupré et al.: Cosmic ray induced ionisation of a molecular cloud shocked by the W28 supernova remnant

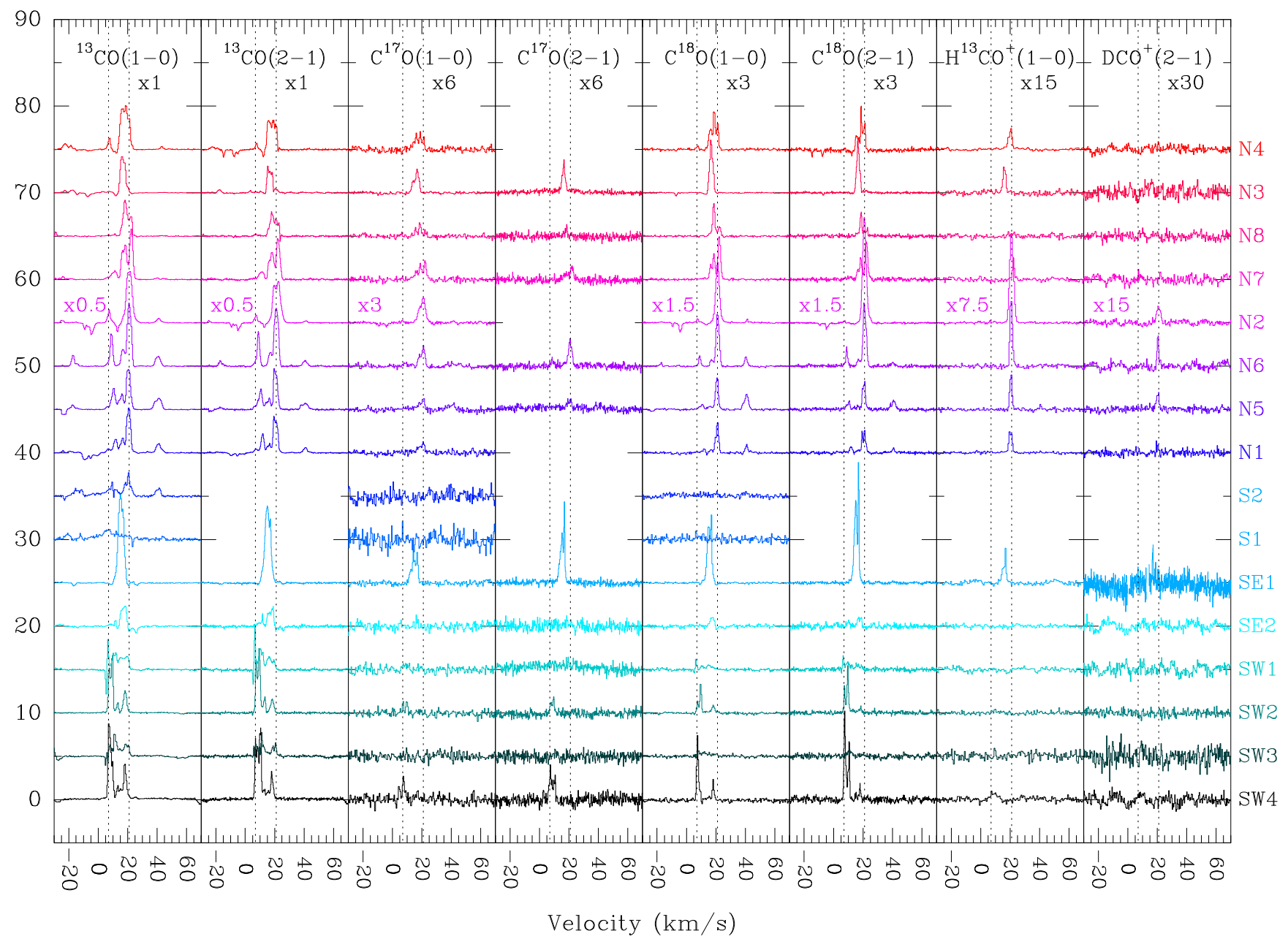

Fig. 2. Observations of millimetre emission lines towards all positions. The intensities are in units of main-beam temperature (K). For readibility, a multiplicative factor was applied to the spectra and is given under each transition line label. This factor was decreased for position N2 (protostar) where the signal is very strong. Vertical dashed lines indicate the two extreme velocity components of the complex at 7 and $21 \mathrm{~km} \mathrm{~s}^{-1}$.

from the $\mathrm{CO}$ observations (Table 5). We derived the observed $\mathrm{DCO}^{+} / \mathrm{HCO}^{+}$abundance ratio for each set of physical conditions $\left(n_{\mathrm{H}_{2}}, T\right)$, assuming ${ }^{12} \mathrm{C} /{ }^{13} \mathrm{C}=50$ (see Sect. 4.2). The uncertainty in the abundance ratio is dominated by the uncertainties in the physical conditions. Results are listed in Table 5 and will be used in the next section to constrain the CR ionisation rate.

\section{Methods to measure the $\mathrm{CR}$ ionisation rate $\zeta$}

\subsection{Analytical method}

In their seminal paper, Guélin et al. (1977, hereafter G77) suggested that the abundance ratio of $\mathrm{DCO}^{+}$to $\mathrm{HCO}^{+}$, which we denote $R_{\mathrm{D}}=\mathrm{DCO}^{+} / \mathrm{HCO}^{+}$, can be used to measure the ionisation fraction in molecular clouds, $x_{\mathrm{e}}=n\left(\mathrm{e}^{-}\right) / n_{\mathrm{H}}$. Subsequently, Caselli et al. (1998, hereafter C98) proposed using the $R_{\mathrm{H}}=\mathrm{HCO}^{+} / \mathrm{CO}$ abundance ratio in combination with $R_{\mathrm{D}}$ to derive both $x_{\mathrm{e}}$ and $\zeta$ in dark clouds. The basic idea is that $\mathrm{DCO}^{+}$and $\mathrm{HCO}^{+}$are chemically linked, and depend on a reduced number of chemical reactions where the $\mathrm{CR}$ ionisation rate plays a crucial role, through the ionisation of $\mathrm{H}_{2}$ into $\mathrm{H}_{2}^{+}$ (Herbst \& Klemperer 1973), leading to the formation of the pivotal $\mathrm{H}_{3}^{+}$ion. The fast ion-neutral reaction of $\mathrm{H}_{3}^{+}$with $\mathrm{HD}$ produces the deuterated ion $\mathrm{H}_{2} \mathrm{D}^{+}$which then initiates the formation of several deuterated species, including $\mathrm{DCO}^{+}$. In a similar fashion, $\mathrm{HCO}^{+}$is formed by the reaction of $\mathrm{H}_{3}^{+}$with $\mathrm{CO}$. This forms the basis of the method of $\mathrm{C} 98$ which uses $\mathrm{CO}, \mathrm{DCO}^{+}$, and $\mathrm{HCO}^{+}$to derive $x_{\mathrm{e}}$ and $\zeta$. The full set of reactions used in the C98 analysis is given in Table A.1 with updated reaction rates.

The steady-state abundance ratios $R_{\mathrm{H}}=\mathrm{HCO}^{+} / \mathrm{CO}$ and $R_{\mathrm{D}}=\mathrm{DCO}^{+} / \mathrm{HCO}^{+}$can be analytically derived from this network, provided that $\mathrm{HCO}^{+}$and $\mathrm{DCO}^{+}$are predominantly formed and destroyed by reactions $1-4$ and 7-9, respectively. One then finds that

$R_{\mathrm{H}}=\frac{\left[\mathrm{HCO}^{+}\right]}{[\mathrm{CO}]}=\frac{k_{\mathrm{H}}}{\beta^{\prime}} \frac{x\left(\mathrm{H}_{3}^{+}\right)}{x_{\mathrm{e}}} \approx \frac{k_{\mathrm{H}}}{\left(2 \beta x_{\mathrm{e}}+\delta\right) \beta^{\prime}} \frac{\zeta / n_{\mathrm{H}}}{x_{\mathrm{e}}}$,

and

$R_{\mathrm{D}}=\frac{\left[\mathrm{DCO}^{+}\right]}{\left[\mathrm{HCO}^{+}\right]} \approx \frac{1}{3} \frac{x\left(\mathrm{H}_{2} \mathrm{D}^{+}\right)}{x\left(\mathrm{H}_{3}^{+}\right)} \approx \frac{1}{3} \frac{k_{\mathrm{f}} x(\mathrm{HD})}{k_{\mathrm{e}} x_{\mathrm{e}}+\delta+k_{\mathrm{f}}^{-1} / 2}$

where $n(X)$ is the number density of species $X$ and $x(X)=n(X) / n_{\mathrm{H}}$ its fractional abundance. In the following, we will assume that the gas is fully molecular such that $n_{\mathrm{H}}=$ $2 n\left(\mathrm{H}_{2}\right)$. The coefficients $\beta, \beta^{\prime}$, and $k$ are the reaction rates listed in Table A.1. Finally, $\delta \sim \delta_{\mathrm{H}_{3}^{+}} \sim \delta_{\mathrm{H}_{2} \mathrm{D}^{+}}$is the total destruction rate of $\mathrm{H}_{3}^{+}$or $\mathrm{H}_{2} \mathrm{D}^{+}$by neutrals. Provided that $R_{\mathrm{H}}, R_{\mathrm{D}}, n_{\mathrm{H}}$, and the kinetic temperature are known, $x_{\mathrm{e}}$ and $\zeta$ can then be derived 
Table 3. Results from the Gaussian fits of the emission lines of ${ }^{13} \mathrm{CO}$ and $\mathrm{C}^{18} \mathrm{O}$ towards the 12 positions where they are detected.

\begin{tabular}{|c|c|c|c|c|c|c|c|c|c|c|c|c|c|c|c|c|}
\hline \multirow[t]{2}{*}{ Pos. } & \multicolumn{4}{|c|}{${ }^{13} \mathrm{CO}(1-0)$} & \multicolumn{4}{|c|}{${ }^{13} \mathrm{CO}(2-1)$} & \multicolumn{4}{|c|}{$\mathrm{C}^{18} \mathrm{O}(1-0)$} & \multicolumn{4}{|c|}{$\mathrm{C}^{18} \mathrm{O}(2-1)$} \\
\hline & $v_{0}$ & W & $T_{\text {peak }}$ & $\Delta v$ & $v_{0}$ & W & $T_{\text {peak }}$ & $\Delta v$ & $v_{0}$ & W & $T_{\text {peak }}$ & $\Delta v$ & $v_{0}$ & W & $T_{\text {peak }}$ & $\Delta v$ \\
\hline \multicolumn{17}{|c|}{ First dominant velocity component } \\
\hline N1 & 20.7 & 17.4 & 5.3 & 3.1 & 20.5 & 15.1 & 4.1 & 3.5 & 20.9 & 2.7 & 1.0 & 2.4 & 21.1 & 1.5 & 0.8 & 1.9 \\
\hline N5 & 20.6 & 15.7 & 5.0 & 3.0 & 20.5 & 15.0 & 4.7 & 3.0 & 20.8 & 2.8 & 1.3 & 2.1 & 20.7 & 2.6 & 1.0 & 2.4 \\
\hline N6 & 21.2 & 23.9 & 7.4 & 3.0 & 21.1 & 24.2 & 7.0 & 3.3 & 21.2 & 5.5 & 2.0 & 2.5 & 21.1 & 6.2 & 2.3 & 2.5 \\
\hline $\mathrm{N} 2$ & 21.1 & 59.6 & 12.6 & 4.5 & 21.5 & 57.4 & 9.6 & 5.6 & 21.1 & 14.7 & 4.5 & 3.0 & 21.0 & 25.0 & 7.9 & 3.0 \\
\hline N7 & 22.5 & 15.3 & 5.8 & 2.5 & 22.4 & 13.9 & 4.8 & 2.7 & 22.3 & 3.6 & 1.7 & 1.9 & 22.3 & 3.4 & 1.5 & 2.1 \\
\hline N8 & 18.4 & 15.4 & 4.0 & 3.6 & 18.2 & 9.7 & 2.5 & 3.6 & 18.6 & 2.4 & 1.3 & 1.7 & 18.6 & 1.3 & 0.9 & 1.4 \\
\hline N3 & 16.8 & 17.3 & 4.4 & 3.7 & 16.6 & 11.6 & 2.8 & 3.9 & 16.8 & 5.3 & 1.9 & 2.6 & 16.7 & 4.6 & 1.9 & 2.3 \\
\hline N4 & 18.9 & 15.2 & 4.9 & 2.9 & 19.0 & 11.3 & 3.3 & 3.2 & 18.9 & 2.2 & 1.5 & 1.4 & 18.8 & 1.4 & 1.6 & 0.8 \\
\hline SE1 & 15.0 & 34.3 & 9.9 & 3.3 & 15.1 & 39.7 & 8.7 & 4.3 & 15.3 & 6.7 & 2.1 & 3.0 & 15.0 & 7.5 & 3.0 & 2.4 \\
\hline SE2 & 16.3 & 5.8 & 2.0 & 2.7 & 16.3 & 5.6 & 1.6 & 3.2 & 16.9 & 0.8 & 0.3 & 2.5 & 16.9 & 0.6 & 0.3 & 1.9 \\
\hline SW2 & 9.6 & 10.5 & 7.0 & 1.4 & 9.5 & 13.2 & 7.4 & 1.7 & 9.7 & 1.3 & 1.1 & 1.1 & 9.7 & 2.2 & 1.8 & 1.2 \\
\hline SW4 & 8.9 & 14.7 & 4.7 & 3.0 & 7.8 & 18.6 & 6.4 & 2.7 & 7.5 & 3.4 & 2.4 & 1.4 & 7.6 & 4.6 & 3.1 & 1.4 \\
\hline \multicolumn{17}{|c|}{ Second dominant velocity component } \\
\hline N1 & 11.7 & 5.6 & 1.5 & 3.6 & 11.8 & 5.7 & 2.2 & 2.5 & 11.9 & 0.3 & 0.2 & 1.8 & 11.9 & 0.5 & 0.2 & 2.1 \\
\hline N5 & 10.4 & 8.0 & 2.3 & 3.3 & 10.4 & 7.3 & 2.1 & 3.3 & 10.4 & 0.6 & 0.2 & 3.2 & 10.1 & 0.8 & 0.3 & 3.0 \\
\hline N6 & 9.0 & 8.3 & 3.7 & 2.1 & 9.0 & 8.1 & 3.9 & 2.0 & 9.0 & 0.6 & 0.4 & 1.5 & 9.0 & 1.0 & 0.7 & 1.4 \\
\hline N7 & 18.6 & 10.0 & 3.9 & 2.4 & 18.3 & 9.1 & 3.2 & 2.7 & 18.7 & 1.8 & 1.0 & 1.8 & 18.6 & 1.6 & 0.8 & 1.9 \\
\hline N8 & 22.3 & 4.5 & 2.2 & 1.9 & 21.3 & 3.0 & 1.6 & 1.8 & 22.3 & 0.9 & 0.3 & 2.3 & 22.1 & 0.8 & 0.2 & 3.3 \\
\hline N4 & 16.0 & 12.4 & 4.5 & 2.6 & 15.8 & 8.3 & 3.4 & 2.3 & 16.3 & 2.3 & 0.8 & 2.6 & 16.1 & 1.5 & 0.5 & 2.5 \\
\hline SE1 & 17.3 & 10.4 & 4.6 & 2.1 & 17.2 & 1.9 & 2.6 & 0.7 & 17.0 & 1.6 & 1.9 & 0.8 & 17.0 & 3.0 & 4.3 & 0.7 \\
\hline SE2 & 18.6 & 4.3 & 2.2 & 1.9 & 18.8 & 3.0 & 1.9 & 1.5 & 18.5 & 0.3 & 0.2 & 1.5 & 18.8 & 0.4 & 0.3 & 1.1 \\
\hline SW2 & 7.6 & 9.1 & 5.3 & 1.6 & 7.5 & 8.0 & 6.2 & 1.2 & 7.6 & 0.6 & 0.4 & 1.3 & 7.5 & 1.0 & 1.0 & 0.9 \\
\hline SW4 & 7.3 & 11.8 & 6.9 & 1.6 & 10.4 & 12.7 & 7.9 & 1.5 & - & - & - & - & 10.5 & 2.5 & 1.9 & 1.2 \\
\hline
\end{tabular}

Notes. The fit parameters are: the centre line velocity $v_{0}$ (in $\mathrm{km} \mathrm{s}^{-1}$, in the local standard of rest), the integrated intensity $W$ (in $\mathrm{K} \mathrm{km} \mathrm{s}^{-1}$ ), the peak temperature $T_{\text {peak }}$ (in $\mathrm{K}$ ), and the full width at half maximum $\Delta v$ (in $\mathrm{km} \mathrm{s}^{-1}$ ). In case of non-detections, upper limits are given at the $1 \sigma$ level. Uncertainties are dominated by calibration $(\sim 20 \%)$. Integrated intensities and peak temperatures are given on the main-beam temperature scale.

Table 4. Results from the Gaussian fits of the emission lines of $\mathrm{H}^{13} \mathrm{CO}^{+}$ and $\mathrm{DCO}^{+}$towards the nine positions where $\mathrm{H}^{13} \mathrm{CO}^{+}$is detected.

\begin{tabular}{lrrrrrrrr}
\hline \hline Pos. & \multicolumn{4}{c}{$\mathrm{H}^{13} \mathrm{CO}^{+}(1-0)$} & \multicolumn{4}{c}{$\mathrm{DCO}^{+}(2-1)$} \\
& $v_{0}$ & $W$ & $T_{\text {peak }}$ & $\Delta v$ & $v_{0}$ & $W$ & $T_{\text {peak }}$ & $\Delta v$ \\
\hline N1 & 20.3 & 0.44 & 0.17 & 2.5 & - & $<0.01$ & $<0.01$ & 3.0 \\
N5 & 20.6 & 0.53 & 0.27 & 1.8 & 20.4 & 0.15 & 0.06 & 2.3 \\
N6 & 20.8 & 1.11 & 0.53 & 2.0 & 20.5 & 0.16 & 0.12 & 1.2 \\
N2 & 21.0 & 4.39 & 1.38 & 3.0 & 21.0 & 0.41 & 0.12 & 3.1 \\
N7 & 22.3 & 0.23 & 0.16 & 1.3 & - & $<0.01$ & $<0.01$ & 3.0 \\
N3 & 16.2 & 0.49 & 0.20 & 2.3 & - & $<0.02$ & $<0.02$ & 3.0 \\
N4 & 20.2 & 0.47 & 0.14 & 3.0 & - & $<0.01$ & $<0.01$ & 3.0 \\
SE1 & 17.0 & 0.15 & 0.23 & 0.6 & 17.0 & 0.13 & 0.16 & 0.76 \\
SW4 & 9.3 & 0.25 & 0.05 & 5.2 & - & $<0.02$ & $<0.02$ & 3.0 \\
\hline
\end{tabular}

Notes. The fit parameters are the same as Table 3. In case of nondetections, upper limits are given at the $1 \sigma$ level. Uncertainties are dominated by calibration $(\sim 20 \%)$. Integrated intensities and peak temperatures are given on the main-beam temperature scale.

as

$x_{\mathrm{e}}=\left(\frac{k_{\mathrm{f}} x(\mathrm{HD})}{3 R_{\mathrm{D}}}-\delta-\frac{k_{\mathrm{f}}}{2} \mathrm{e}^{-\Delta E / T}\right) \frac{1}{k_{e}}$,

$\zeta / n_{\mathrm{H}}=\frac{\beta^{\prime}}{k_{\mathrm{H}}}\left(2 \beta x_{\mathrm{e}}+\delta\right) R_{\mathrm{H}} x_{\mathrm{e}}$

where $\Delta E=220 \mathrm{~K}$, such that at sufficiently low temperatures the last term in brackets in Eq. (4) becomes negligible. Equation (4) demonstrates that $x_{\mathrm{e}}$ only depends on the abundance ratio $R_{\mathrm{D}}$ and the gas kinetic temperature, as originally proposed by G77.
Figure 3 shows $x_{\mathrm{e}}$ as a function of $R_{\mathrm{D}}$, as predicted from Eq. (4), assuming a kinetic temperature of $20 \mathrm{~K}$ (blue dashed line). We note two regimes in the dependence of $x_{\mathrm{e}}$ on $R_{\mathrm{D}}$. For low $R_{\mathrm{D}}$ values $\left(\lesssim 10^{-2}\right), x_{\mathrm{e}}$ is proportional to $1 / R_{\mathrm{D}}$ with a factor that depends on the chemical reaction rates and the HD abundance. For higher $R_{\mathrm{D}}$ values, $x_{\mathrm{e}}$ drops sharply. In this regime, $x_{\mathrm{e}}$ varies by more than two orders of magnitude when $R_{\mathrm{D}}$ is changed by only a factor of two. The slope becomes steeper with increasing temperature, due to the predominance of the reverse reaction of the formation of $\mathrm{H}_{2} \mathrm{D}^{+}$(Table A.1). This indicates that accurate values of $x_{\mathrm{e}}$ in dark clouds through this method require extremely accurate values of $R_{\mathrm{D}}$. It also suggests that in regions with higher ionisation, where $x_{\mathrm{e}}$ is proportional to $1 / R_{\mathrm{D}}$, $\mathrm{DCO}^{+}$will be difficult to detect.

\subsection{Numerical models}

To assess the validity of the analytical approach of G77 and C98, we have solved the OSU $2009^{2}$ chemical network for the abundances of $\mathrm{DCO}^{+}, \mathrm{HCO}^{+}$, and $\mathrm{e}^{-}$, using the astrochem ${ }^{3}$ code. The time evolution of the gas-phase abundances was followed until a steady state was reached, after typically $10 \mathrm{Myr}$. The underlying hypothesis is that the cloud was already molecular when it was irradiated by the CRs emitted at the SN explosion. The chemical changes caused by the sudden CR irradiation are dominated by ion-neutral reactions, whose timescale is only $\sim 10^{2} \mathrm{yr}$, much shorter than the W28 SNR age $\left(\sim 10^{4} \mathrm{yr}\right.$; see Introduction). Details of the models are given in Appendix A. In

\footnotetext{
2 http://www physics.ohio-state.edu/ eric/research. html

3 http://smaret.github.io/astrochem/
} 
Table 5. Physical conditions and cosmic ray ionisation rates.

\begin{tabular}{|c|c|c|c|c|c|c|c|c|c|}
\hline Pos. & $\begin{array}{c}\Delta v \\
{\left[\mathrm{~km} \mathrm{~s}^{-1}\right]}\end{array}$ & $\begin{array}{r}n_{\mathrm{H}_{2}} \\
{\left[10^{3} \mathrm{~cm}^{-3}\right]}\end{array}$ & $\begin{array}{l}T_{k i n} \\
{[\mathrm{~K}]}\end{array}$ & $\begin{array}{r}N\left(\mathrm{C}^{18} \mathrm{O}\right) \\
{\left[10^{15} \mathrm{~cm}^{-2}\right]}\end{array}$ & $\begin{array}{r}A_{V} \\
{[\mathrm{mag}]}\end{array}$ & $\begin{array}{l}N\left(\mathrm{H}^{13} \mathrm{CO}^{+}\right) \\
{\left[10^{12} \mathrm{~cm}^{-2}\right]}\end{array}$ & $\begin{array}{r}N\left(\mathrm{DCO}^{+}\right) \\
{\left[10^{12} \mathrm{~cm}^{-2}\right]}\end{array}$ & $R_{\mathrm{D}}=\frac{\left[\mathrm{DCO}^{+}\right]}{\left[\mathrm{HCO}^{+}\right]}$ & $\begin{array}{r}\zeta \\
{\left[10^{-17} \mathrm{~s}^{-1}\right]}\end{array}$ \\
\hline N1 & 3.5 & $0.6\{0.2-1\}$ & $15 \pm 5$ & $4\{2-6\}$ & $21\{11-32\}$ & $0.8-1.3$ & $<0.22$ & $<0.005$ & $>13$ \\
\hline N5 & 3.0 & $4\{2-5\}$ & $10 \pm 2$ & $3\{2-8\}$ & $16\{11-32\}$ & $1.1-1.4$ & $0.89-1.30$ & $0.014-0.020$ & $130-330$ \\
\hline N6 & 3.0 & $4\{2-6\}$ & $13 \pm 3$ & $6\{4-20\}$ & $32\{21-105\}$ & $1.8-2.5$ & $0.79-1.30$ & $0.008-0.012$ & $130-400$ \\
\hline $\mathrm{N} 2^{\dagger}$ & 5.0 & $>2$ & $16 \pm 2$ & $20\{15-30\}$ & $105\{79-158\}$ & $5.6-8.9$ & $1.10-2.00$ & $0.003-0.006$ & - \\
\hline N7 & 2.5 & $2\{2-5\}$ & $10 \pm 2$ & $4\{3-10\}$ & $21\{16-53\}$ & $0.6-0.9$ & $<0.25$ & $<0.007$ & $>130$ \\
\hline N8 & 3.5 & $1\{0.6-2\}$ & $8 \pm 1$ & $3\{2-4\}$ & $16\{11-21\}$ & $<0.2$ & $<0.35$ & - & - \\
\hline N3 & 3.5 & $6\{4-10\}$ & $8 \pm 1$ & $6\{5-7\}$ & $32\{26-37\}$ & $1.0-1.4$ & $<0.35$ & $<0.006$ & $>260$ \\
\hline N4 & 3.0 & $2\{0.6-4\}$ & $12 \pm 3$ & $2\{2-3\}$ & $11\{5-16\}$ & $1.0-1.4$ & $<0.35$ & $<0.006$ & $>40$ \\
\hline SE1 & 4.0 & $2\{1-5\}$ & $19 \pm 5$ & $6\{5-20\}$ & $32\{26-105\}$ & $0.4-0.56$ & $0.79-1.0$ & $0.032-0.05$ & $0.2-20$ \\
\hline SE2 & 3.0 & $4\{2-10\}$ & $8 \pm 2$ & $0.9\{0.4-20\}$ & $5\{2-105\}$ & $<0.2$ & $<0.28$ & - & - \\
\hline SW2 & 1.5 & $2\{1-4\}$ & $20 \pm 4$ & $4\{3-10\}$ & $21\{16-53\}$ & $<0.1$ & $<0.22$ & - & - \\
\hline $\mathrm{SW} 4^{\dagger}$ & 1.5 & $6\{4-10\}$ & $16 \pm 2$ & $1.5\{1-3\}$ & $5\{5-16\}$ & $0.5-0.8$ & $<0.25$ & $<0.009$ & - \\
\hline
\end{tabular}

Notes. $n_{\mathrm{H}_{2}}$ is the molecular hydrogen density $\left(\mathrm{cm}^{-3}\right), T_{\text {kin }}$ the gas kinetic temperature, $N\left(\mathrm{C}^{18} \mathrm{O}\right)$ the total column density of $\mathrm{C}^{18} \mathrm{O} . A_{V}$ is the visual extinction assuming $\left[\mathrm{C}^{18} \mathrm{O}\right]=A_{V} \times 1.910^{14} \mathrm{~cm}^{-2}$ (Frerking et al. 1982; Bolatto et al. 2013). We assumed isotopic ratio values ${ }^{18} \mathrm{O} /{ }^{16} \mathrm{O}=500$ and ${ }^{13} \mathrm{C} /{ }^{12} \mathrm{C}=50$ (see text). Values in brackets indicate the range of values satisfying $\chi_{v}^{2}<1$. Uncertainties on $n_{\mathrm{H}_{2}}$ and $T_{\text {kin }}$ are at the $70 \%$ confidence level, and are propagated in the abundance ratios and upper limits. Lower limits of $\zeta$ were deduced from chemical modelling (see Sect. 6). ${ }^{(\dagger)}$ N2 and SW4 are probably ionised by a source other than CRs (see text).

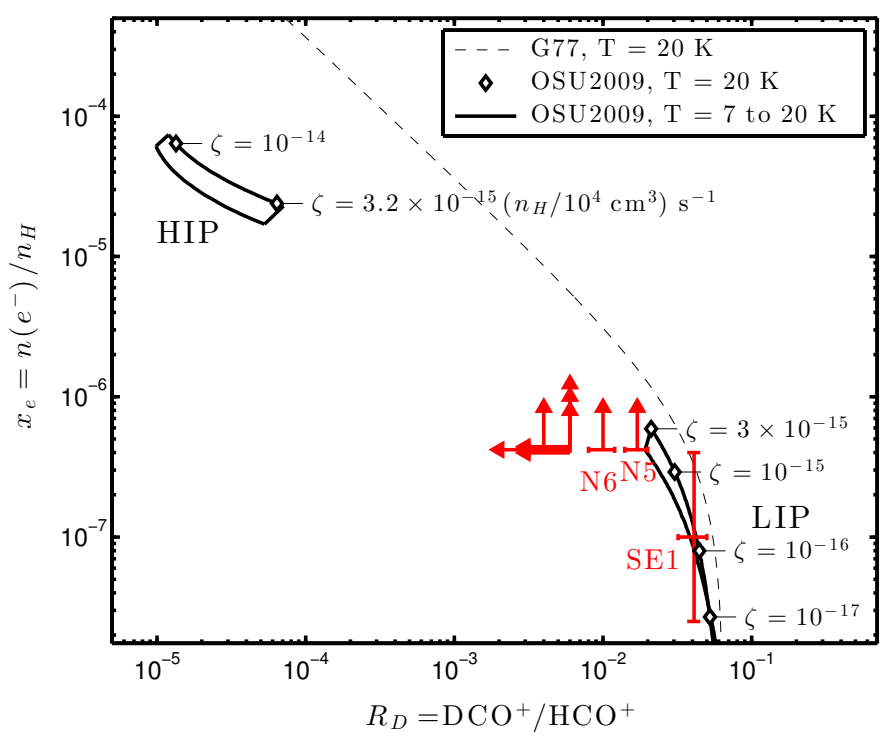

Fig. 3. $x_{\mathrm{e}}=n\left(\mathrm{e}^{-}\right) / n_{\mathrm{H}}$ as a function of $R_{\mathrm{D}}$. Values from the analytical method (G77) in Sect. 5.1 are given at $T=20 \mathrm{~K}$ (dashed line). Results from our calculation (see Sect. 5.2) for temperatures between $7 \mathrm{~K}$ and $20 \mathrm{~K}$ are contained within the solid lines. $\zeta$ values are given for $n_{\mathrm{H}}=10^{4} \mathrm{~cm}^{-3}$, although the model only depends on the $\zeta / n_{\mathrm{H}}$ ratio (see text). $R_{\mathrm{D}}$ values or upper limits as derived from observations are indicated by the red symbols; see Sect. 6.1.

one calculation, the gas is shielded by 20 mag of visual extinction, such that UV photons can safely be ignored.

As anticipated from Eq. (2), we found that the abundances mostly depend on the $\zeta / n_{\mathrm{H}}$ ratio, rather than on $\zeta$ and $n_{\mathrm{H}}$ separately. This behaviour is similar to photon-dominated regions in which a good parameter is the ratio of the UV radiation field to the total density. For each temperature from $7 \mathrm{~K}$ to $20 \mathrm{~K}$, a series of calculations with $\zeta / n_{\mathrm{H}}$ increasing from $10^{-22} \mathrm{~cm}^{3} \mathrm{~s}^{-1}$ to $10^{-18} \mathrm{~cm}^{3} \mathrm{~s}^{-1}$ were performed, and the steady-state values of $x_{\mathrm{e}}$ and $R_{\mathrm{D}}$ were recorded.

In these calculations, we assumed a standard $\mathrm{CO}$ abundance of $\sim 7.3 \times 10^{-5}$ in the cloud. Indeed, since the density of the studied clouds is relatively low (Table 5), we do not expect $\mathrm{CO}$ depletion to play an important role here. However, in general, one has to take into account this uncertainty. In addition, our calculations do not consider separately the $\mathrm{H} 2$ ortho-to-para ratio, which is known to affect the $\mathrm{DCO}^{+} / \mathrm{HCO}^{+}$abundance ratio when it is larger than about 0.1 (e.g. Pagani et al. 2011). Our choice here is based on the published observations that indicate that the $\mathrm{H} 2$ ortho-to-para ratio is smaller than about 0.01 in molecular clouds (e.g Troscompt et al. 2009; Dislaire et al. 2012).

\subsection{A new view of the $\mathrm{DCO}+/ \mathrm{HCO}^{+}$method}

The results of the numerical models are shown in Fig. 3. As expected, the ionisation fraction $x_{\mathrm{e}}$ increases with $\zeta / n_{\mathrm{H}}$, with $R_{\mathrm{D}}$ decreasing in the process. There is good overall agreement between the analytical and numerical predictions for $R_{\mathrm{D}} \gtrsim$ $2 \times 10^{-2}$. In this high- $R_{\mathrm{D}}$ regime, the small differences between analytical and numerical values are due to the abundances of HD and CO not being constant as originally assumed by G77 and C98. Instead, as $\zeta / n_{\mathrm{H}}$ and $x_{\mathrm{e}}$ increase, atomic deuterium becomes more abundant. Similarly, the CO abundance decreases because of the dissociating action of CRs. When $R_{\mathrm{D}}$ decreases and reaches $\approx 2 \times 10^{-2}$, the abundances predicted by the numerical model change dramatically to a regime characterised by large values of $x_{\mathrm{e}}$ and low values of $R_{\mathrm{D}}$. This jump corresponds to the well-known transition from the so-called low ionisation phase (LIP) to the high ionisation phase (HIP) (Pineau des Forêts et al. 1992; Le Bourlot et al. 1993), and is due to the sensitivity of interstellar chemical networks to ionisation. The LIP is associated with $R_{\mathrm{D}}$ larger than $10^{-2}$, whilst the HIP is characterised by $R_{\mathrm{D}} \lesssim 10^{-4}$. In our calculations, the LIP-HIP transition occurs at $\zeta / n_{\mathrm{H}} \sim 3 \times 10^{-19} \mathrm{~cm}^{3} \mathrm{~s}^{-1}$, and we note that this value depends only slightly on the temperature, although it is known to depend on other parameters such as the gas-phase abundance of metals (Wakelam et al. 2006a). A detailed analysis of the LIP-HIP transition is, however, not the aim of this study. Here, it is rather the existence of this instability which is of interest since it produces a sharp difference between the analytical and the numerical predictions from the ionisation point of view. Application to a practical case shows that what changes is not the jump itself but 
(slightly) the $\zeta$ at which it occurs (see e.g. Ceccarelli et al. 2011). In the former, the variations of $x_{\mathrm{e}}$ and $R_{\mathrm{D}}$ are continuous and, as already mentioned, predict $x_{\mathrm{e}} \sim 1 / R_{\mathrm{D}}$ in the low- $R_{\mathrm{D}}$ regime. This scaling is, however, not observed in the numerical models, and is replaced by a discontinuous variation of both $x_{\mathrm{e}}$ and $R_{\mathrm{D}}$. The present calculations show that the LIP is characterised by $R_{\mathrm{D}}=10^{-2}-10^{-1}, x_{\mathrm{e}} \lesssim 5 \times 10^{-7}$, and the HIP is characterised by $R_{\mathrm{D}} \approx$ few $10^{-5}$ and $x_{\mathrm{e}} \approx$ few $10^{-5}$.

As shown in Fig. 4, the low values of $R_{\mathrm{D}}$ in the HIP are due to a very low abundance of $\mathrm{DCO}^{+}$, whilst the abundance of $\mathrm{HCO}^{+}$ decreases by a smaller amount. This has important consequences when using the $\mathrm{DCO}^{+} / \mathrm{HCO}^{+}$method to derive the ionisation fraction and $\mathrm{CR}$ ionisation rate. First, it must be recognised that this method may provide a value of $x_{\mathrm{e}}$ only for LIP-dominated gas conditions. In other words, where $\mathrm{DCO}^{+}$is detected, the line of sight is dominated by low- $x_{\mathrm{e}}$ gas. For lines of sight dominated by HIP gas, the abundance of $\mathrm{DCO}^{+}$is expected to be well below detectable thresholds, such that only upper limits on $R_{\mathrm{D}}$ can be derived. Yet, an upper limit on $R_{\mathrm{D}}$ still provides essential information, since it is associated with a lower limit on $x_{\mathrm{e}}$, which in turn corresponds to a lower limit of $\zeta / n_{\mathrm{H}}$. On the contrary, for LIP-dominated lines of sight, the value of $x_{\mathrm{e}}$ and $\zeta / n_{\mathrm{H}}$ may be derived directly from $R_{\mathrm{D}}$, although $x_{\mathrm{e}}$ is extremely sensitive to uncertainties on $R_{\mathrm{D}}$ in this regime.

\section{The $C R$ ionisation rate in W28}

\subsection{Constant density and temperature cloud analysis}

A new view of the $\mathrm{DCO}^{+} / \mathrm{HCO}^{+}$method thus emerges, which stresses its strengths and limitations. The method allows the determination of the ionisation fraction $x_{\mathrm{e}}$ and the $\zeta / n_{\mathrm{H}}$ ratio for gas in the LIP, and provides lower limits of $x_{\mathrm{e}}$ and $\zeta / n_{\mathrm{H}}$ for gas in the HIP. In the following, we apply this method to the sample of observed points, using the constraints on the gas temperature and density, and the $R_{\mathrm{D}}$ value in each point (Table 5). We emphasise that the model calculations summarised in Fig. 3 assumed constant density and gas temperature. In the next section, we will discuss how the $\mathrm{DCO}^{+} / \mathrm{HCO}^{+}$method can be used to constrain $x_{\mathrm{e}}$ and $\zeta / n_{\mathrm{H}}$, taking into account the thermal structure of the cloud.

Of the 16 lines of sight initially observed in $\mathrm{CO}, 12$ were also detected in $\mathrm{H}^{13} \mathrm{CO}^{+}$, of which 4 led to $R_{\mathrm{D}}$ determinations and 5 to upper limits (Table 5). The four points with measured $R_{\mathrm{D}}$ are N5, N6, SE1, and N2. In the following analysis, we exclude N2 as it coincides with a protostar, which means that a more accurate analysis taking into account the structure of the protostar and the inner ionisation is necessary. The values obtained towards N5, N6, and SE1 are shown in Fig. 3.

The SE1 point lies on the LIP branch, enabling a determination of the ionisation fraction $x_{\mathrm{e}}=(0.15-4) \times 10^{-7}$ and of the $\mathrm{CR}$ ionisation rate $\zeta=(0.2-20) \times 10^{-17} \mathrm{~s}^{-1}$. On the contrary, the values of $R_{\mathrm{D}}$ towards N5 and N6 lie in the gap between the LIP and HIP branches, even when considering a kinetic temperature as high as $20 \mathrm{~K}$, a temperature which is larger than the values derived for these positions. In these cases, adopting $n_{\mathrm{H}}=2 n\left(\mathrm{H}_{2}\right) \gtrsim 4 \times 10^{3} \mathrm{~cm}^{-3}$ (Table 5), Fig. 3 provides the following lower limits: $x_{\mathrm{e}} \gtrsim 4 \times 10^{-7}$ and $\zeta \gtrsim 1.3 \times 10^{-15} \mathrm{~s}^{-1}$ for both points. We note that the detection of $\mathrm{DCO}^{+}$indicates that the line of sight includes a non-negligible amount of LIP, which can serve to further constrain the value of $\zeta / n_{\mathrm{H}}$. This can be seen in Fig. 4, which shows $R_{\mathrm{D}}$ and $\mathrm{DCO}^{+}$as a function of $\zeta$, for a density and temperature appropriate to position N5 (Table 5). The measured $R_{\mathrm{D}}$ intersects the model predictions at the edge of

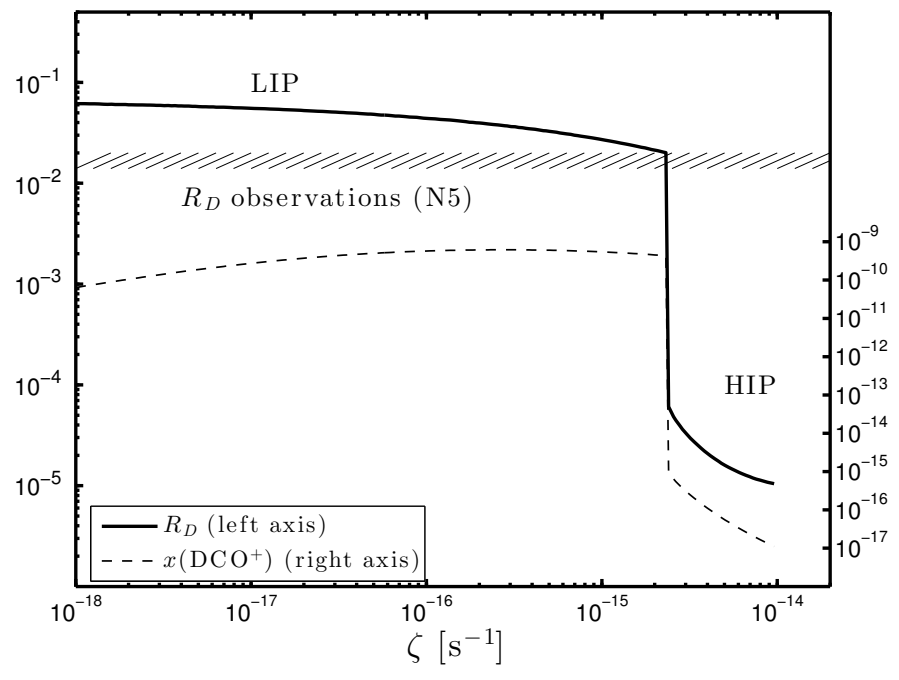

Fig. 4. $R_{\mathrm{D}}=\mathrm{DCO}^{+} / \mathrm{HCO}^{+}$(thick line, left axis) and $x\left(\mathrm{DCO}^{+}\right)=n\left(\mathrm{DCO}^{+}\right) / n_{\mathrm{H}}$ (dashed line, right axis) as a function of $\zeta$, for $T=10 \mathrm{~K}$ and $n_{\mathrm{H}}=810^{3} \mathrm{~cm}^{-3}$, i.e. the physical conditions characterising position N5. The HIP and LIP are marked. The hatched area shows the range of observed $R_{\mathrm{D}}$ at that position.

the LIP/HIP jump, at $\zeta \approx 2.5 \times 10^{-15} \mathrm{~s}^{-1}$. More importantly, the figure shows that the gas is neither entirely in the LIP nor HIP state as expected from the detection of $\mathrm{DCO}^{+}$. A similar plot has also been obtained for N6, leading to the same conclusion.

Finally, the non-detection of $\mathrm{DCO}^{+}$in the other lines of sight leads to upper limits on $R_{\mathrm{D}}$ that are well outside the LIP branch. At these positions, the gas is very likely to be almost entirely in the HIP state, which means that $\zeta / n_{\mathrm{H}} \gtrsim 3 \times 10^{-19} \mathrm{~cm}^{3} \mathrm{~s}^{-1}$. An exception is the point SW4, where an extremely energetic outflow has been detected (Harvey \& Forveille 1988), and where the $\mathrm{HCO}^{+}$is therefore likely contaminated by the outflowing material.

\subsection{Constant density cloud analysis}

As discussed in the previous section, the points N5 and N6 are likely composed of a mixture of gas in the LIP and HIP state. This is similar to the situation observed in W51C-E (CC2011). In that case, $\zeta$ was estimated with a model that takes into account the thermal and chemical structure of a constant density cloud, where a fraction (the deepest) is in the LIP and the rest in the HIP (Fig. 2 in CC2011). Here, we do a similar analysis, using basic arguments instead of a sophisticated model, and we show that it leads to similar results, namely the determination of $\zeta$ to within a factor of 2 . The advantage of this analysis is that it shows in a straightforward way the uncertainty due to the model parameters.

The crucial point is understanding what causes the gas to flip from the HIP to the LIP state going deeper into the cloud. Since the column density is too low to appreciably reduce $\zeta$ across the cloud, the only macroscopic quantity that changes is the gas temperature. Specifically, the temperature increases by a few $\mathrm{K}$ (in the UV-shielded region) going deeper into the cloud because the CO line opacity increases and, consequently, the line cooling becomes less efficient. The effect is larger for larger $\zeta$ as the heating, dominated by the CR ionisation, is less compensated by the line cooling.

It is instructive to see how the $R_{\mathrm{D}}$ ratio changes as a function of the gas temperature for different $\zeta$. This is shown 


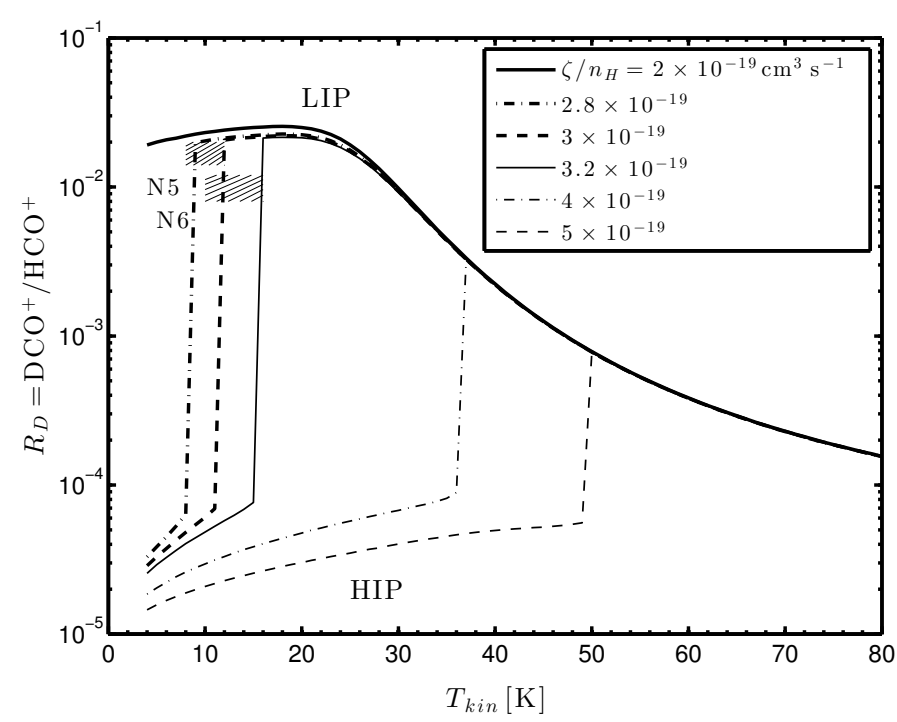

Fig.5. $R_{\mathrm{D}}$ as a function of the gas temperature $T_{\text {kin }}$ for different values of $\zeta / n_{\mathrm{H}}$ : from 2 to $5 \times 10^{-19} \mathrm{~s}^{-1}$, as marked. Note that for $\zeta / n_{\mathrm{H}} \leq 2 \times 10^{-19} \mathrm{~cm}^{3} \mathrm{~s}^{-1}$ (thick solid line), the cloud is always in the LIP, regardless of the temperature. For $\zeta / n_{\mathrm{H}}>5 \times 10^{-19} \mathrm{~cm}^{3} \mathrm{~s}^{-1}$ (thin dashed curve), the cloud is always in the HIP for temperatures $\leq 50 \mathrm{~K}$. Hatched areas show observations of N5 and N6. We assume $A_{V}=20 \mathrm{mag}$.

in Fig. 5, for a range of temperatures $(5-80 \mathrm{~K})$ and $\zeta / n_{\mathrm{H}}$ (2-5 $\times 10^{-19} \mathrm{~cm}^{3} \mathrm{~s}^{-1}$, appropriate to the N5 and N6 points). In these calculations, we consider a cell of gas of constant density, shielded by $20 \mathrm{mag}$ of visual extinctions as before, such that the ionisation is driven by the CRs. The figure shows important features:

i) for $\zeta / n_{\mathrm{H}} \lesssim 2 \times 10^{-19} \mathrm{~cm}^{3} \mathrm{~s}^{-1}$, the cloud is always in the LIP, regardless of the temperature;

ii) for $\zeta / n_{\mathrm{H}} \gtrsim 5 \times 10^{-19} \mathrm{~cm}^{3} \mathrm{~s}^{-1}$, the cloud is always in the HIP for temperatures lower than $50 \mathrm{~K}$;

iii) for intermediate values of $\zeta / n_{\mathrm{H}}$, the gas flips from HIP to LIP with increasing temperature, and the larger $\zeta$ is, the larger the temperature where the flip occurs.

These calculations show that there is a range of ionisation rates in which the gas is extremely sensitive to temperature variations. A tiny increase in temperature is sufficient to make the gas flip from the HIP to the LIP. In particular, for position N5, there is such a combination of values of $R_{\mathrm{D}}, T_{\text {kin }}$, and $\zeta / n_{\mathrm{H}}$ (see Fig. 5) that places it precisely in a region where the transition from HIP to LIP can be triggered by an increase in $T_{\text {kin }}$ as small as a few $\mathrm{K}$. A similar argument applies to N6. In addition, in regions exposed to an enhanced CR ionisation rate, the outer part characterised by large ionisation fractions will be extended farther into the cloud, thus decreasing the relative amount of LIP with respect to the HIP.

Based on the derived kinetic temperatures and values of $R_{\mathrm{D}}$ (Table 5) and using Fig. 5, we can further constrain the value of $\zeta / n_{\mathrm{H}}$. Towards $\mathrm{N} 5$, the temperature was found to be $10 \pm 2 \mathrm{~K}$, while $R_{\mathrm{D}}=0.014-0.020$. When inserted into Fig. 5 , these delineate a region that is compatible with a narrow range of $\zeta / n_{\mathrm{H}}=(2.8-3.0) \times 10^{-19} \mathrm{~cm}^{3} \mathrm{~s}^{-1}$. For the N6 line of sight, we find similar values, $(2.9-3.2) \times 10^{-19} \mathrm{~cm}^{3} \mathrm{~s}^{-1}$. The densities derived from the analysis and their uncertainties then lead to cosmic ray ionisation rates of $(1.3-3.3) \times 10^{-15} \mathrm{~s}^{-1}$ and $(1.3-4.0) \times 10^{-15} \mathrm{~s}^{-1}$ for N5 and N6 respectively. Results are summarised in Table 5 and are included in Figs. 6 and 7.

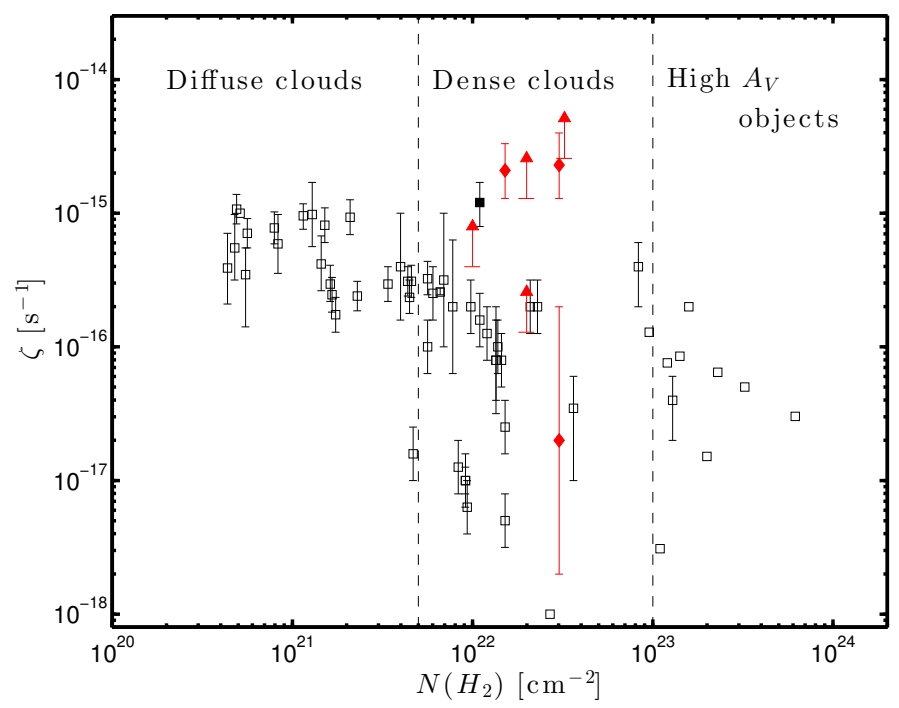

Fig. 6. Compilation of measured $\zeta$ in different objects (open squares), as reported by Padovani \& Galli (2013). The black filled square denotes W51 (Ceccarelli et al. 2011). Red points and lower limits report the values derived in this work. The dashed lines show the range of column densities $(0.5-10) \times 10^{22} \mathrm{~cm}^{-2}$, typical of dense molecular clouds, corresponding to visual extinctions of 5 and $100 \mathrm{mag}$, respectively. On the left lie the diffuse clouds and on the right highly obscured environments such as infrared dark clouds or protoplanetary discs.

\section{Discussion}

Table 5 lists the observed positions and the corresponding CR ionisation rates derived using the method described in the previous section. With the exception of the SE1 point, in all other points $\zeta$ is at least 10 to 260 times larger than the standard value $\left(1 \times 10^{-17} \mathrm{~s}^{-1}\right)$ in Galactic clouds. This is shown in Fig. 6, where we present a compilation of the $\zeta$ measured in various objects (from Padovani \& Galli 2013), plus our measurements. In the range of column densities $(0.5-10) \times 10^{22} \mathrm{~cm}^{-2}$, typical of dense molecular clouds, the points in which we derived $\zeta$ are those with the highest values, together with the CC2011 point (filled square). The first conclusion of this work is, therefore, that clouds next to SNR are indeed irradiated by an enhanced flux of CRs of relatively low energy (see below for a more quantitative statement on the CR particle energies).

The dependence of $\zeta$ on the projected distance from the SNR radio boundary (assuming a W28 distance of $2 \mathrm{kpc}$ ) is shown in Fig. 7. Remarkably, the point farthest $(\sim 10 \mathrm{pc})$ from the SNR edge is the one with the lowest $\zeta$. Actually, it is the only point where the gas is predominantly in the LIP state. All other points, at distances $\lesssim 3 \mathrm{pc}$, have at least a fraction of the gas in the HIP, namely they have a larger $x_{\mathrm{e}}$ and $\zeta$. Of course, this analysis does not take into account the 3D structure of the SNR complex. Yet, this can still provide us with constraints on the propagation properties of CRs, as will be discussed in the following.

Valuable additional information is provided by observations in the $\gamma$-ray domain. Both the northern and southern clouds coincide with sources of TeV emission, as seen by HESS. This means that the clouds are illuminated by very high energy $(\gtrsim 10 \mathrm{TeV})$ CRs, which already escaped the SNR expanding shell and travelled the $\gtrsim 10 \mathrm{pc}$ (or more, if projection effects play a role) to the southern cloud. Conversely, the low CR ionisation rate measured in SE1 tells us that the ionising lower energy CRs remain confined closer to the SNR. In the same vein, GeV emission has been detected towards the northern region but only towards a 


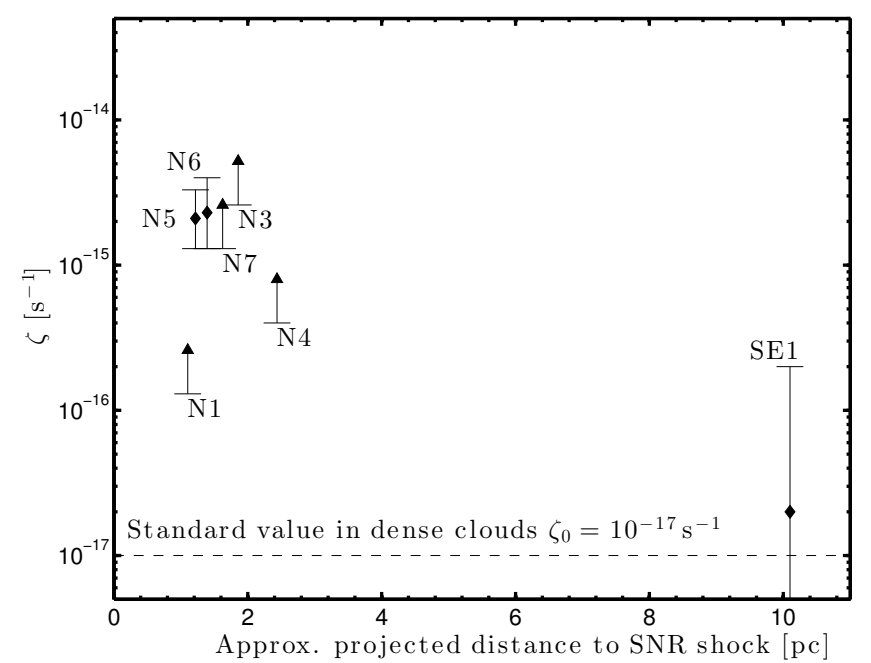

Fig. 7. CR ionisation rate $\zeta$ as a function of the approximate projected distance from the SNR radio boundary (blue circle in Fig. 1), assuming a W28 distance of 2 pkc. We note that the $\zeta$ error bars are dominated by the uncertainties on the $\mathrm{H}_{2}$ densities (see text).

part of the southern one. This difference between the $\mathrm{GeV}$ and $\mathrm{TeV} \gamma$-ray morphology has been interpreted as a projection effect: the portion of the southern region that exhibits a lack of $\mathrm{GeV}$ emission is probably located at a distance from the shock significantly larger than the projected one, $>10 \mathrm{pc}$, and thus can be reached by $\gtrsim \mathrm{TeV}$ CRs but not by $\gtrsim \mathrm{GeV}$ ones (Gabici et al. 2010; Li \& Chen 2010; Nava \& Gabici 2013). Remarkably, the $\mathrm{SE} 1$ point is located in the region where the lack of $\mathrm{GeV}$ emission is observed.

The picture that emerges is that of a stratified structure with CRs of larger and larger energies occupying larger and larger volumes ahead of the shock. Within this framework, it is possible to estimate the CR diffusion coefficient in the region. This can be done by recalling that in a given time $t$, CRs diffuse over a distance $R \sim \sqrt{D \times t}$, where $D$ is the energy dependent diffusion coefficient. For the situation under examination, one gets

$D_{(\approx 10 \mathrm{GeV})} \gtrsim 3 \times 10^{27}\left(\frac{R}{10 \mathrm{pc}}\right)^{2}\left(\frac{t}{10^{4} \mathrm{yr}}\right)^{-1} \mathrm{~cm}^{2} / \mathrm{s}$,

where $D_{(\approx 10 \mathrm{GeV})}$ is the diffusion coefficient of $\approx 10 \mathrm{GeV} \mathrm{CRs,}$ which are those responsible for the $\approx \mathrm{GeV} \gamma$-ray emission, and $t$ is the time elapsed since the escape of CRs from the SNR. The value obtained in Eq. (6) is in substantial agreement with more accurate studies (see e.g. Nava \& Gabici 2013).

The diffusion coefficient obtained in Eq. (6) can then be rescaled to lower energies, according to $D \propto p^{s} \beta$, where $p$ is the particle momentum, $\beta=v / c$ its velocity in units of the speed of light, and $s$ depends on the spectrum of the ambient magnetic turbulence. The typical value of $s$ in the interstellar medium is poorly constrained to be in the range 0.3 to 0.7 (Castellina \& Donato 2011). In the following, we adopt $s=0.5$. To estimate the diffusion length of low energy CRs, one has to keep in mind that, while CRs with energies above $\approx \mathrm{GeV}$ are virtually free from energy losses (the energy loss time for proton-proton interactions in a density $n_{\mathrm{H}} \approx 10^{3} \mathrm{~cm}^{-3}$ is comparable to the age of the SNR), lower energy CRs suffer severe ionisation losses over a short timescale (Berezinskii et al. 1990):

$\tau_{\text {ion }} \approx 14\left(\frac{n_{\mathrm{H}}}{10^{3} \mathrm{~cm}^{-3}}\right)^{-1}\left(\frac{E}{\mathrm{MeV}}\right)^{3 / 2} \mathrm{yr}$.

This approximate expression is sufficiently accurate in the range of energies spanning 1-100 MeV. The diffusion length of low energy CRs can then be estimated by equating the diffusion time $\tau_{\mathrm{d}} \sim R_{\mathrm{d}}^{2} / D$ to the energy loss time $\tau_{\text {ion }}$, which gives $R_{\mathrm{d}} \approx 0.02$, 0.3 , and $3 \mathrm{pc}$ for CRs of energy 1,10 , and $100 \mathrm{MeV}$, respectively. This implies that only CRs with energies $\gtrsim 100 \mathrm{MeV}$ can escape the shock and spread over a distance of $3 \mathrm{pc}$ or more, and thus these are the CRs that play a major role in ionising the gas. Whether the ionisation of the gas is due directly to these CRs or to the products of their interaction with the gas (namely slowed down lower energy CRs) remains an open question. It is remarkable that the particle energies of ionising CRs $(\approx 0.1-1 \mathrm{GeV})$ also make them capable of producing sub- $\mathrm{GeV} \gamma$ rays, given that the kinetic energy threshold for $\pi^{0}$ production is $\approx 280 \mathrm{MeV}$.

Of course, the order of magnitude estimates discussed in this section cannot substitute in any way more sophisticated calculations, yet they clearly indicate an intriguing possible link between low and high energy observations of SNR environments. In fact, in the scenario described above, the very same CRs are responsible for both ionisation of the gas and production of low energy $\gamma$ rays. If confirmed, such a link would constitute robust evidence for the presence of accelerated protons in the environment of the SNR W28, a thing that would bring further support to the idea that SNR are the sources of Galactic CRs. Additional theoretical investigations are needed in order to examine and possibly rule out alternative scenarios which may include other contributions to the ionisation rate (e.g. CR electrons, X-ray photons) or different means of propagation (e.g. straight-line or advective propagation of low energy CRs).

\section{Conclusion}

In this work, we presented new observations to measure the CR ionisation rate in molecular clouds close to supernova remnants (SNR). In doing so, the $\mathrm{DCO}^{+} / \mathrm{HCO}^{+}$method was also revisited. The major results may be summarised as follows.

1) We observed the two lowest rotational transitions of ${ }^{13} \mathrm{CO}$ and $\mathrm{C}^{18} \mathrm{O}$ towards 16 positions in the northern and southern clouds close to the SNR W28. The four lines were detected in emission towards 12 of these positions, where we could, therefore, derive the physical conditions using a nonLTE LVG analysis. With the exception of one position (N2) coinciding with a protostar in the region, we derived $\mathrm{H}_{2}$ densities and temperatures typical of molecular clouds, namely $n_{\mathrm{H}_{2}}=(0.2-10) \times 10^{3} \mathrm{~cm}^{-3}$ and $T=6-24 \mathrm{~K}$. We searched for $\mathrm{H}^{13} \mathrm{CO}^{+}$and $\mathrm{DCO}^{+}$line emission in the above 12 positions, and detected it in 9 and 4 , respectively. From these data, we could derive the $R_{\mathrm{D}}=\mathrm{DCO}^{+} / \mathrm{HCO}^{+}$in 4 positions, one of which coincides with the protostar, and give upper limits for the remaining 5 positions.

2) We reinvestigated the $\mathrm{DCO}^{+} / \mathrm{HCO}^{+}$method used to derive the ionisation fraction $x_{\mathrm{e}}=n\left(\mathrm{e}^{-}\right) / n_{\mathrm{H}}$ and the relevant CR ionisation rate $\zeta$ causing it. To this aim, we compared the steady-state abundances of $\mathrm{HCO}^{+}, \mathrm{DCO}^{+}$, and $\mathrm{e}^{-}$as predicted by the analytical model of $\mathrm{G} 77$, to numerical calculations, assuming constant density and gas temperature. The numerical model leads to two well separated regimes of ionisation, also known as the low- and high-ionisation phases (LIP and HIP; Pineau des Forêts et al. 1992). In the context of this work, these two phases lead to two separated regimes in terms of $\zeta$ and $R_{\mathrm{D}}$ values:

i) for $\zeta / n_{\mathrm{H}} \lesssim 3 \times 10^{-19} \mathrm{~cm}^{3} \mathrm{~s}^{-1}$, the gas is in the LIP, where $R_{\mathrm{D}} \gtrsim 2 \times 10^{-2}$ and $x_{\mathrm{e}} \lesssim 6 \times 10^{-7}$. In this regime, the dependence of $x_{\mathrm{e}}$ on $R_{\mathrm{D}}$ is very steep leading to large uncertainties on $x_{\mathrm{e}}$; 
ii) for $\zeta / n_{\mathrm{H}} \gtrsim 3 \times 10^{-19} \mathrm{~cm}^{3} \mathrm{~s}^{-1}$, the gas is in the HIP, where $R_{\mathrm{D}} \lesssim 10^{-4}$ and $x_{\mathrm{e}} \gtrsim 2 \times 10^{-5}$. In this regime, $\mathrm{DCO}^{+}$is not detectable and the numerical prediction for $x_{\mathrm{e}}$ differs significantly from the analytical one.

Therefore, the $\mathrm{DCO}^{+} / \mathrm{HCO}^{+}$abundance ratio can provide a measure of $x_{\mathrm{e}}$ and $\zeta$ in the LIP, and only lower limits if the gas is in the HIP.

3) We found only one position, SE1, in the LIP, where $\quad R_{\mathrm{D}}=0.032-0.05, x_{\mathrm{e}}=(0.3-4) \times 10^{-7} \quad$ and $\zeta=(0.2-20) \times 10^{-17} \mathrm{~s}^{-1}$. Two positions, N5 and N6, lie in the gap between the LIP and HIP, namely the gas is neither entirely in the LIP nor in the HIP, although it certainly contains a fraction of gas in the LIP, where $\mathrm{DCO}^{+}$ is detectable (and detected). The jump from the HIP to the LIP when penetrating farther into the cloud is associated with an increase in the temperature, and we showed that model calculations at several temperatures further constrain the value of $\zeta$. The uncertainty in $\zeta$ towards these positions is dominated by the uncertainty in the $\mathrm{H}_{2}$ density and the derived values are $\zeta=(1.3-3.3)$ and $(1.3-4.0) \times 10^{-15} \mathrm{~s}^{-1}$ for N5 and N6, respectively. Towards the remaining 5 positions with upper limits on $R_{\mathrm{D}}$, the derived $\zeta$ values are at least 10 to 260 times higher than the standard value of $1 \times 10^{-17} \mathrm{~s}^{-1}$.

4) The points of the northern cloud have the largest CR ionisation rates measured so far in the Galaxy. The point towards the southern cloud is, on the contrary, consistent with the average galactic CR ionisation rate of molecular clouds not interacting with a SNR. Since the northern and southern clouds have projected distances from the SNR shock of $\leq 3$ and $\sim 10 \mathrm{pc}$, respectively, this can be explained by the fact that the low energy ionising CRs have not reached the southern cloud yet. On the other hand, the observations show that both the northern and southern clouds coincide with $\mathrm{TeV}$ emission sources, suggesting that high $\gtrsim 10 \mathrm{TeV}$ CRs have reached both. This is also consistent with $\gamma$-ray emission sources inciding with the northern cloud but only partially with the southern cloud, indicating that the former is irradiated by $\approx 0.1-1 \mathrm{GeV}$ CRs, while only the nearest portion of the southern cloud is so affected.

5) The emerging picture is that of energy-dependent diffusion properties of hadronic CRs. The high-energy CRs responsible for $\mathrm{TeV} \gamma$-ray emission through $\pi^{0}$-decay can diffuse far ahead of the SNR shock, while the low-energy CRs $(0.1-1 \mathrm{GeV})$, responsible for both the low $\gamma$-ray emission and the ionisation of the gas, remain closer to the SNR shock. The present work thus gives first observational evidence to the theoretical predictions that hadrons of energy $0.1-1 \mathrm{GeV}$ contribute most to the ionisation in dense gas (Padovani et al. 2009).

Acknowledgements. We warmly thank Marco Padovani for providing us with his compilation and for useful discussions. This work has been financially supported by the Programme National Hautes Énergies (PNHE). Based on observations carried out with the IRAM $30 \mathrm{~m}$ telescope. IRAM is supported by INSU/CNRS (France), MPG (Germany) and IGN (Spain). S. Gabici acknowledges the financial support of the UnivEarthS Labex Program at Sorbonne Paris Cité (ANR-10LABX-0023 and ANR-11-IDEX-0005-02).

\section{Appendix A: Chemical models}

We solved the OSU $2009^{4}$ chemical network using the astrochem ${ }^{5}$ code.

\footnotetext{
4 http://www.physics.ohio-state.edu/ eric/research. html

5 http://smaret.github.io/astrochem/
}

Table A.1. Reduced chemical network for the analytical derivation of $\mathrm{DCO}^{+} / \mathrm{HCO}^{+}$.

\begin{tabular}{|c|c|c|c|c|}
\hline \multicolumn{4}{|c|}{ Reaction } & \multirow{2}{*}{$\begin{array}{l}\text { Reaction rate }\left[\mathrm{cm}^{3} \mathrm{~s}^{-1}\right] \\
\zeta\left[\mathrm{s}^{-1}\right]\end{array}$} \\
\hline No. 1 & $\mathrm{CR}+\mathrm{H}_{2}$ & $\stackrel{\zeta}{\rightarrow}$ & $\mathrm{H}_{2}^{+}+\mathrm{e}^{-}$ & \\
\hline No. 2 & $\mathrm{H}_{2}^{+}+\mathrm{H}_{2}$ & $\stackrel{k_{\mathrm{H}_{2}^{+}}}{\rightarrow}$ & $\mathrm{H}_{3}^{+}+\mathrm{H}$ & $k_{\mathrm{H}_{2}^{+}}=2.110^{-9}$ \\
\hline No. 3 & $\mathrm{H}_{3}^{+}+\mathrm{CO}$ & $\stackrel{k_{\mathrm{H}}}{\rightarrow}$ & $\mathrm{HCO}^{+}+\mathrm{H}_{2}$ & $k_{\mathrm{H}}=1.6110^{-9}$ \\
\hline No. 4 & $\mathrm{HCO}^{+}+\mathrm{e}^{-}$ & $\stackrel{\beta^{\prime}}{\rightarrow}$ & $\mathrm{CO}+\mathrm{H}$ & $\beta^{\prime}=2.810^{-7}\left(\frac{T}{300}\right)^{-0.69}$ \\
\hline \multirow[t]{2}{*}{ No. 5} & $\mathrm{H}_{3}^{+}+\mathrm{e}^{-}$ & $\stackrel{\beta}{\rightarrow}$ & $\mathrm{H}+\mathrm{H}+\mathrm{H}$ & $\beta=4.3610^{-8}\left(\frac{T}{300}\right)^{-0.52}$ \\
\hline & & & $\mathrm{H}_{2}+\mathrm{H}$ & $+2.3410^{-8}\left(\frac{T}{300}\right)^{-0.52}$ \\
\hline No. 6 & $\mathrm{H}+\mathrm{H}$ & $\stackrel{k^{\prime}}{\rightarrow}$ & $\mathrm{H}_{2}$ & $k^{\prime}=4.9510^{-17}\left(\frac{T}{300}\right)^{0.50}$ \\
\hline \multirow[t]{2}{*}{ No. 7} & $\mathrm{H}_{3}^{+}+\mathrm{HD}$ & $\underset{k_{\mathrm{c}}^{-1}}{\stackrel{k_{\mathrm{f}}}{\rightleftharpoons}}$ & $\mathrm{H}_{2} \mathrm{D}^{+}+\mathrm{H}_{2}$ & $k_{\mathrm{f}}=1.710^{-9}$ \\
\hline & & & & $k_{\mathrm{f}}^{-1}=k_{\mathrm{f}} \mathrm{e}^{-220 / T}$ \\
\hline No. 8 & $\mathrm{H}_{2} \mathrm{D}^{+}+\mathrm{CO}$ & $\stackrel{k_{\mathrm{D}}}{\rightarrow}$ & $\mathrm{DCO}^{+}+\mathrm{H}_{2}$ & $k_{\mathrm{D}}=5.3710^{-10}$ \\
\hline No. 9 & $\mathrm{DCO}^{+}+\mathrm{e}^{-}$ & $\stackrel{\beta^{\prime}}{\rightarrow}$ & $\mathrm{CO}+\mathrm{D}$ & $\beta^{\prime}=2.810^{-7}\left(\frac{T}{300}\right)^{-0.69}$ \\
\hline \multirow[t]{3}{*}{ No. 10} & $\mathrm{H}_{2} \mathrm{D}^{+}+\mathrm{e}^{-}$ & $\stackrel{k_{e}}{\rightarrow}$ & $\mathrm{H}+\mathrm{H}+\mathrm{D}$ & $k_{\mathrm{e}}=4.3810^{-8}\left(\frac{T}{300}\right)^{-0.50}$ \\
\hline & & & $\mathrm{H}_{2}+\mathrm{D}$ & $+1.2010^{-8}\left(\frac{T}{300}\right)^{-0.50}$ \\
\hline & & & $\mathrm{HD}+\mathrm{H}$ & $+4.2010^{-9}\left(\frac{T}{300}\right)^{-0.50}$ \\
\hline No. 11 & $H+D$ & $\stackrel{k^{\prime \prime}}{\rightarrow}$ & $\mathrm{HD}$ & $k^{\prime \prime}=\sqrt{2} k^{\prime}$ \\
\hline No. 12 & $\mathrm{H}_{2} \mathrm{D}^{+}+\mathrm{CO}$ & $\stackrel{k_{\mathrm{D}}^{\prime}}{\rightarrow}$ & $\mathrm{HCO}^{+}+\mathrm{H}_{2}$ & $k_{\mathrm{D}}^{\prime}=1.110^{-9}$ \\
\hline \multirow[t]{2}{*}{ No. 13} & $\mathrm{H}_{3}^{+}+\mathrm{D}$ & $\underset{k^{\prime-1}}{\stackrel{k_{f}^{\prime}}{\rightleftharpoons}}$ & $\mathrm{H}_{2} \mathrm{D}^{+}+\mathrm{H}$ & $k_{\mathrm{f}}^{\prime}=1.010^{-9}$ \\
\hline & & & & $k_{\mathrm{f}}^{\prime-1}=k_{\mathrm{f}}^{\prime} \mathrm{e}^{-632 / T}$ \\
\hline No. 14 & $\mathrm{CO}^{+}+\mathrm{HD}$ & $\stackrel{k}{\mathrm{CO}^{+}} \rightarrow$ & $\mathrm{DCO}^{+}+\mathrm{H}$ & $k_{\mathrm{CO}^{+}}=7.510^{-10}$ \\
\hline
\end{tabular}

Notes. The reduced network corresponds to the original description by Guélin et al. (1977) and Caselli et al. (1998). The rates of reactions 1-6 are contained in the original OSU 2009 network. We appended deuterated reactions 7-14 for which chemical rates are taken from Roberts \& Millar (2000). Reaction 14 is only dominant in the HIP and is not involved in the analytical determination of $\mathrm{DCO}^{+} / \mathrm{HCO}^{+}$.

Table A.2. Range of initial physical parameters used in the astrochem code.

\begin{tabular}{cc}
\hline \hline Parameter & Range \\
\hline$A_{V}$ & $20 \mathrm{mag}$ \\
$n_{\mathrm{H}}$ & $10^{3}$ to $10^{4} \mathrm{~cm}^{-3}$ \\
$T_{\text {kin }}$ & 5 to $80 \mathrm{~K}$ \\
$T_{\mathrm{d}}$ & $20 \mathrm{~K}$ \\
$\zeta$ & $10^{-18}$ to $10^{-14} \mathrm{~s}^{-1}$ \\
\hline
\end{tabular}

astrochem is a numerical code that computes the timedependent chemical abundances in a cell of gas shielded by a given visual extinction $A_{V}$ and with given physical parameters: the total $\mathrm{H}$ density $n_{\mathrm{H}}$, the gas kinetic temperature $T_{\text {kin }}$, and the dust temperature $T_{\mathrm{d}}$. It also takes as an input the initial chemical abundances and the CR ionisation rate. We followed the abundance until a steady state was reached, for a grid of models covering a large range of physical conditions $\left(n_{\mathrm{H}}, T_{\mathrm{kin}}\right)$ and $\mathrm{CR}$ ionisation rates $\zeta$, at a given $A_{V}=20 \mathrm{mag}$, far inside the cloud, where the gas is shielded from the UV radiation field and the ionisation is dominated by CRs. The results only depend on the $\zeta / n_{\mathrm{H}}$ ratio (see Sect. 5.2). The role of the dust in astrochem is limited to the absorption and desorption processes, namely no grain surface chemistry is considered. As discussed in Sect. 5.2, neither process is relevant to the present discussion. Initial conditions were taken from the low-metal abundances as in Graedel et al. (1982) and Wakelam et al. (2006b) using an updated $\mathrm{He} / \mathrm{H}$ 
relative abundance of 0.09 (Asplund et al. 2009). In Table A.2, we list the range of physical parameters used in this study.

The OSU network contains 6046 reactions involving 468 species. We appended 12 reactions labeled 7-14 in Table A.1 involving the deuterated species: $\mathrm{D}, \mathrm{HD}, \mathrm{H}_{2} \mathrm{D}^{+}$, and $\mathrm{DCO}^{+}$. Chemical rates are taken from Roberts \& Millar (2000).

\section{References}

Abdo, A. A., Ackermann, M., Ajello, M., et al. 2010, ApJ, 718, 348 Abdo, A. A., Ackermann, M., Ajello, M., et al. 2011, ApJ, 734, 28 Ackermann, M., Ajello, M., Allafort, A., et al. 2013, Science, 339, 807 Aharonian, F. A. 2013, Astropart. Phys., 43, 71

Aharonian, F., Akhperjanian, A. G., Bazer-Bachi, A. R., et al. 2008, A\&A, 481, 401

Arikawa, Y., Tatematsu, K., Sekimoto, Y., \& Takahashi, T. 1999, PASJ, 51, L7 Asplund, M., Grevesse, N., Sauval, A. J., \& Scott, P. 2009, ARA\&A, 47, 481 Balbus, S. A., \& Hawley, J. F. 1998, Rev. Mod. Phys., 70, 1 Bell, A. R. 1978, MNRAS, 182, 147

Berezinskii, V. S., Bulanov, S. V., Dogiel, V. A., \& Ptuskin, V. S. 1990, Cosmic ray astrophysics (Amsterdam: North Holland)

Boger, G. I., \& Sternberg, A. 2006, ApJ, 645, 314

Bolatto, A. D., Wolfire, M., \& Leroy, A. K. 2013, ARA\&A, 51, 207

Brogan, C. L., Gelfand, J. D., Gaensler, B. M., Kassim, N. E., \& Lazio, T. J. W. 2006, ApJ, 639, L25

Caselli, P., Walmsley, C. M., Terzieva, R., \& Herbst, E. 1998, ApJ, 499, 234

Castellina, A., \& Donato, F. 2011, Planets, Stars and Stella Systems (SpringerVerlag) [arXiv: 1110.2981]

Ceccarelli, C., Maret, S., Tielens, A. G. G. M., Castets, A., \& Caux, E. 2003, A\&A, 410, 587

Ceccarelli, C., Hily-Blant, P., Montmerle, T., et al. 2011, ApJ, 740, L4

Claussen, M. J., Frail, D. A., Goss, W. M., \& Gaume, R. A. 1997, ApJ, 489, 143

Dame, T. M., Hartmann, D., \& Thaddeus, P. 2001, ApJ, 547, 792

Dislaire, V., Hily-Blant, P., Faure, A., et al. 2012, A\&A, 537, A20

Elmegreen, B. G. 1998, ASP Conf. Ser., 148, 150

Federman, S. R., Lambert, D. L., Sheffer, Y., et al. 2003, ApJ, 591, 986

Ferrière, K. M. 2001, Rev. Mod. Phys., 73, 1031

Flower, D. R. 1999, MNRAS, 305, 651

Frail, D. A., Goss, W. M., \& Slysh, V. I. 1994, ApJ, 424, L111

Frerking, M. A., Langer, W. D., \& Wilson, R. W. 1982, ApJ, 262, 590

Fukui, Y., Sano, H., Sato, J., et al. 2012a, ARA\&A, 746, 82

Fukui, Y., Sano, H., Sato, J., et al. 2012b, ApJ, 746, 82

Gabici, S., Casanova, S., Aharonian, F. A., \& Rowell, G. 2010, in SF2A2010: Proc. of the Annual meeting of the French Society of Astronomy and Astrophysics, eds. S. Boissier, M. Heydari-Malayeri, R. Samadi, \& D. VallsGabaud, 313

Giuliani, A., Tavani, M., Bulgarelli, A., et al. 2010, A\&A, 516, L11

Goudis, C. 1976, Ap\&SS, 45, 133

Graedel, T. E., Langer, W. D., \& Frerking, M. A. 1982, ApJS, 48, 321

Guélin, M., Langer, W. D., Snell, R. L., \& Wootten, H. A. 1977, ApJ, 217, L165
Harvey, P. M., \& Forveille, T. 1988, A\&A, 197, L19

Herbst, E., \& Klemperer, W. 1973, ApJ, 185, 505

Hewitt, J. W., Yusef-Zadeh, F., \& Wardle, M. 2008, ARA\&A, 683, 189

Hillas, A. M. 2005, J. Phys. G Nucl. Phys., 31, 95

Ilovaisky, S. A., \& Lequeux, J. 1972, A\&A, 18, 169

Indriolo, N., \& McCall, B. J. 2012, ApJ, 745, 91

Indriolo, N., Blake, G. A., Goto, M., et al. 2010, ApJ, 724, 1357

Kaspi, V. M., Lyne, A. G., Manchester, R. N., et al. 1993, ApJ, 409, L57

Le Bourlot, J., Pineau des Forêts, G., Roueff, E., \& Schilke, P. 1993, ApJ, 416, L87

Le Bourlot, J., Pineau des Forêts, G., \& Roueff, E. 1995a, A\&A, 297, 251

Le Bourlot, J., Pineau des Forêts, G., Roueff, E., \& Flower, D. R. 1995b, A\&A, 302,870

Lee, H. H., Roueff, E., Pineau des Forêts, G., et al. 1998, A\&A, 334, 1047

Lefloch, B., Cernicharo, J., \& Pardo, J. R. 2008, A\&A, 489, 157

Lesur, G., Kunz, M. W., \& Fromang, S. 2014, A\&A, 566, A56

Li, H., \& Chen, Y. 2010, MNRAS, 409, L35

Linsky, J. L., \& Wood, B. 1995, in Am. Astron. Soc. Meet. Abstr., 27, 1347

Lozinskaya, T. A. 1974, Sov. Astron., 17, 603

Lozinskaya, T. A. 1981, Sov. Astron. Lett., 7, 17

McCall, B. J., Huneycutt, A. J., Saykally, R. J., et al. 2003, Nature, 422, 500

Milam, S. N., Savage, C., Brewster, M. A., Ziurys, L. M., \& Wyckoff, S. 2005 , ApJ, 634, 1126

Milne, D. K., \& Wilson, T. L. 1971, A\&A, 10, 220

Morlino, G., Amato, E., \& Blasi, P. 2009, MNRAS, 392, 240

Nava, L., \& Gabici, S. 2013, MNRAS, 429, 1643

Nicholas, B. P., Rowell, G., Burton, M. G., et al. 2012, ARA\&A, 419, 251

Padovani, M., \& Galli, D. 2013, Astrophys. Space Sci. Proc., 34, 61

Padovani, M., Galli, D., \& Glassgold, A. E. 2009, A\&A, 501, 619

Padovani, M., Hennebelle, P., \& Galli, D. 2013, in SF2A-2013: Proc. of the Annual meeting of the French Society of Astronomy and Astrophysics. eds. L. Cambresy, F. Martins, E. Nuss, \& A. Palacios, 409

Pagani, L., Roueff, E., \& Lesaffre, P. 2011, ApJ, L35

Pety, J. 2005, SF2A-2005: Semaine de l'Astrophysique Francaise (EDP Sciences), Conf. Ser., 721

Pineau des Forêts, G., Roueff, E., \& Flower, D. R. 1992, MNRAS, 258, 45

Reynolds, S. P. 2008, ARA\&A, 46, 89

Rho, J., \& Borkowski, K. J. 2002, ApJ, 575, 201

Roberts, H., \& Millar, T. J. 2000, A\&A, 361, 388

Taquet, V., Ceccarelli, C., \& Kahane, C. 2012, ApJ, 748, L3

Troscompt, N., Faure, A., Maret, S., et al. 2009, A\&A, 506, 1243

van Dishoeck, E. F., \& Black, J. H. 1988, ApJ, 334, 771

Velázquez, P. F., Dubner, G. M., Goss, W. M., \& Green, A. J. 2002, AJ, 124, 2145

Wakelam, V., Herbst, E., \& Selsis, F. 2006a, A\&A, 451, 551

Wakelam, V., Herbst, E., Selsis, F., \& Massacrier, G. 2006b, A\&A, 459, 813

Westerhout, G. 1958, Bull. Astron. Inst., 14, 215

Wilson, T. L., \& Rood, R. 1994, ARA\&A, 32, 191

Wootten, A. 1981, ApJ, 245, 105

Yang, B., Stancil, P. C., Balakrishnan, N., \& Forrey, R. C. 2010, ApJ, 718, 1062

Yusef-Zadeh, F., Shure, M., Wardle, M., \& Kassim, N. 2000, ApJ, 540, 842 